\title{
On pure global dimension of locally finitely presented Grothendieck categories
}

by

Daniel Simson (Toruń)

Abstract. In the paper we define and investigate a pure global dimension of any locally finitely presented Grothendieck category. The problem of the computation of the pure global dimension is reduced to computation of the supremum of projective dimensions of flat objects in a certain functor category. Categories having the pure global dimension zero are characterized. Finally, a characterization of perfect functor categories is given.

In [13] Griffith defines the left pure global dimension for any ring $R$ and, among other things, characterizes commutative rings $R$ with P.gl. $\operatorname{dim} R=0$ as artinian principal ideal rings or, equivalently, as rings $R$ for which every $R$-module is a direct sum of cyclic modules. Pure global dimension of rings has also been investigated by Gruson and Jensen [14], and by Kielpiński and Simson [18].

In the present paper pure global dimension P.gl. dim $\mathscr{A}$ is defined and investigated for an arbitrary locally finitely presented Grothendieck category $\mathscr{A}$. Conditions which reflect finiteness of P.gl.dim $\mathscr{A}$ are given. One of the main results is a characterization of locally finitely presented Grothendieck categories $\mathscr{A}$ with P.gl.dim $\mathscr{A}=0$ (see Theorem 6.3 and [31]) which asserts for instance that P.gl.dim $\mathscr{A}=0$ if and only if $\mathscr{A}$ is pure-perfect, or equivalently, if $\mathscr{A}$ is locally noetherian and every its object is a direct sum of finitely generated subobjects. Moreover, it is shown that 1.P.gl. $\operatorname{dim} R=0$ if and only if the natural homomorphism

$$
\left(\prod Q_{t}\right) \otimes_{R} M \rightarrow \prod\left(Q_{t} \otimes_{R} M\right)
$$

is injective for any family $\left\{Q_{t}\right\}$ of right $R$-modules and any left $R$-module $M$.

Recently Gruson and Jensen have proved that $1 . P . g 1 . \operatorname{dim} R=0=1 . P . g 1 . \operatorname{dim} R$ if and only if $R$ is left artinian and there are only finitely many isomorphy classes of finitely generated indecomposable left $R$-modules. Using this interesting result, we prove the following conjecture of Brauer-Thrall, proved in [27]: Let $A$ be a finitedimensional algebra over a field $k$, and suppose that there are infinitely many isomorphy classes of finite-dimensional indecomposable $A$-modules. Then, for any natural number $n$, there are indecomposable $A$-modules of finite dimension $\geqslant n$.

The main method applied in the paper is the following. Let $L(\mathscr{A})$ be the category 
of all additive contravariant functors from the category $\mathrm{fp}(\mathscr{A})$ of all finitely presented objects of $\mathscr{A}$ to the category Ab of abelian groups, and let $h .: \mathscr{A} \rightarrow L(\mathscr{A})$ be the natural extension of the Yoneda functor. It is observed that there are natural equivalences

$$
\operatorname{Pext}_{\mathscr{A}}^{n}(-, ?)=\operatorname{Ext}_{L(\mathscr{A})}^{n}\left(h_{-}, h_{\eta}\right), \quad n \geqslant 0,
$$

and therefore $\mathscr{A}$ is equivalent to the full subcategory of $L(\mathscr{A})$ consisting of all flat objects. Then it follows that P.gl. $\operatorname{dim} \mathscr{A}=0$ if and only if every flat object in $L(\mathscr{A})$ is projective, i.e., $L(\mathscr{A})$ is a perfect category (see [3], [19] and [39]).

The organization of the paper is as follows. The first two sections contain preliminary results about categories, purity and pure dimensions. Section 3 is devoted to the study of stable and factorizable systems. In particular, a characterization of Mittag-Leffler objects is given (cf. [26]). In Section 4 we prove that any locally finitely presented Grothendieck category has sufficiently many pure-injective objects. Section 5 contains a characterization of coperfect and perfect functor categories. Section 6 is devoted to the study of categories $\mathscr{A}$ with P.gl.dim $\mathscr{A}=0$. The last Section 7 contains some examples.

The paper is partially based on [33]. Some results were announced in [32].

The author would like to thank R. Kiełpiński for many discussions during the preparation of this paper.

§ 1. Preliminaries. A Grothendieck category is an abelian category $\mathscr{A}$ which has a set of generators and admits colimits that are exact when taken over directed sets (cf. [6], [9] and [25]).

Let $\mathscr{A}$ be a Grothendieck category. An object $M$ of $\mathscr{A}$ is of finite type if for each directed increasing family of its subobjects $M_{i}$ with $M=U M_{i}$ there is an $j$ with $M=M_{j} . M$ is finitely presented if it is of finite type and if, for each epimorphism $f: N \rightarrow M$ with $N$ of finite type, the kernel of $f$ is also of finite type. $M$ is noetherian (resp. artinian) if the class of subobjects of $M$ satisfies the ascending (resp. descending) chain condition. Finally, $M$ is finite if it is both artinian and noetherian. $\mathscr{A}$ is locally finitely presented (resp. locally of finite type, ...) if it has a family of finitely presented (resp. finite type, ...) generators.

By an additive category we shall mean a category together with an abelian group structure on each of its Hom sets such that composition is bilinear (cf. [22]).

Let $\mathscr{C}$ be a small additive category. A $\mathscr{C}$-module is a covariant additive functor from $\mathscr{C}$ to the category of abelian groups. The category of all $\mathscr{C}$-modules together with natural transformations of functors as morphisms will be denoted by $\mathscr{G}$-Mod. This is a natural generalization of the notion of module over a ring. $\mathscr{C}$-Mod is a locally finitely presented Grothendieck category and $\mathscr{C}$-modules of the form

$$
h^{X}=\operatorname{Hom}_{\mathscr{C}}(X,-), \quad X \in \mathscr{C},
$$

form a set of its finitely presented projective generators. A $\mathscr{C}$-module $M$ is free if it is isomorphic with a direct sum of modules $h^{X}$. M is finitely generated (resp. cyclic) if it is an epimorphic image of a finite direct sum of the modules $h^{x}$ (resp. of a module $h^{X}$ ). A left ideal in the category $\mathscr{C}$ is a $\mathscr{C}$-submodule of $h^{X}$ for some $X \in \mathscr{C}$, a right ideal in $\mathscr{C}$ is a submodule of a $\mathscr{C}^{\mathrm{op}}$-module $h_{X}=\operatorname{Hom}_{\mathscr{C}}(-, X), X \in \mathscr{C}$. A two-sided ideal in $\mathscr{C}$ is a subfunctor of the functor

$$
\mathrm{Hom}_{\mathscr{C}}: \mathscr{C}^{\mathrm{op}} \times \mathscr{C} \rightarrow \mathrm{Ab} .
$$

If $I$ is a two-sided ideal in $\mathscr{C}$ and $M$ is a $\mathscr{C}$-module $I M$ is a $\mathscr{C}$-submodule of $M$ such that $I M(X), X \in \mathscr{C}$, is a subgroup of $M(X)$ generated by all elements of the form $M(f) y$ with $y \in M(Y)$ and $f \in I(Y, X)$ for some $Y \in \mathscr{C}$. It is clear that the quotient $\mathscr{C}$-module $M / I M$ is, in a natural way, a $\mathscr{C} / I$-module, where the quotient category $\mathscr{C} / I$ has the same objects as $\mathscr{C}$ and

$$
\operatorname{Hom}_{\mathscr{C} I I}(X, Y)=\operatorname{Hom}_{\mathscr{C}}(X, Y) / I(X, Y) \text {. }
$$

The Jacobson radical of an additive category $\mathscr{C}$ is a two-sided ideal $J(\mathscr{C})$ defined by

$J(\mathscr{C})(A, B)=\left\{f \in \operatorname{Hom}_{\mathscr{C}}(A, B), 1_{A^{-}} g f\right.$ has a two-sided inverse for every $\left.g\right\}$ (see [22]). It is not difficult to check that $J(\mathscr{C} / J(\mathscr{C}))=0$ and that $J(\mathscr{C})(X, X)$ is the Jacobson radical of the endomorphism ring

$$
\text { End }_{\mathscr{C}} X=\operatorname{Hom}_{\mathscr{C}}(X, X)
$$

for every object $X$ from $\mathscr{C}$. Moreover, one can prove the following

LEMMA 1.1. Let $M=\oplus_{i=1}^{n} M_{i}$ and let $f=\left(f_{i j}\right): M \rightarrow M$ where $f_{i j}: M_{j} \rightarrow M_{i}$.

Then $f \in J\left(\operatorname{End}_{\mathscr{G}} M\right)$ if and only if $f_{i j} \in J(\mathscr{C})\left(M_{j}, M_{i}\right)$ for all $i$ and $j$.

A small additive category $\mathscr{C}$ is semi-simple if each $\mathscr{C}$-module $h^{X}, X \in \mathscr{C}$, is a coproduct of simple left ideals. $\mathscr{C}$ is regular in the sense of von Neumann if for each of its morphisms $f$ there exists a $g$ such that $f=f g f$.

Proposition 1.2. Let $\mathscr{C}$ be a small additive category. The following conditions are equivalent:

(a) $\mathscr{C}$ is semi-simple.

(b) $\mathscr{C}$ is regular in the sense of von Neumann and the ring $\operatorname{End}_{\mathscr{C}} X$ is semi-simple for every $X \in \mathscr{C}$.

(c) The endomorphism ring of any finitely generated free $\mathscr{C}$-module is semi-simple.

Proof. The implication (a) $\rightarrow$ (c) is obvious.

(c) $\rightarrow$ (b). Let $f: X \rightarrow Y$ be a morphism in $\mathscr{C}$. Consider the element

$$
s=\left(\begin{array}{cc}
0 & 0 \\
h^{f} & 0
\end{array}\right) \in S=\operatorname{End}_{\mathscr{B}-\text { Mod }} h^{X} \oplus h^{Y}
$$

Since $S$ is semi-simple, there exists a $t \in S$ such that $s=s t s$. Hence $f=f g f$ for some $g$ and (b) follows.

Observe that, for every $X \in \mathscr{C}$, there is a one-to-one correspondence between left ideals in $E=\operatorname{End}_{\mathscr{C}} X$ generated by idempotents and direct summands of $h^{X}$ which is given by $E g \mapsto \operatorname{Im} h^{g}$. Therefore (b) $\rightarrow$ (a) and the proof is complete. 
COROLlary 1.3. (a) $\mathscr{C}$ is semi-simple iff $\mathscr{C}^{\mathrm{op}}$ is semi-simple.

(b) If $\mathscr{C}$ has finite coproducts, then it is semi-simple if and only if $\operatorname{End}_{\mathscr{C}} X$ is semisimple for each $X \in \mathscr{C}$.

Recall that the tensor product functor

$$
\otimes_{\mathscr{C}}: \mathscr{C}^{\mathrm{op}}-\mathrm{Mod} \times \mathscr{C}-\mathrm{Mod} \rightarrow \mathrm{Ab}
$$

is defined by

$$
N \otimes_{\mathscr{C}} M=\oplus\left(N X \otimes_{z} M X, X \in \mathscr{C}\right) / U,
$$

where $U$ is. a subgroup of $\oplus\left(N X \otimes_{Z} M X, X \in \mathscr{C}\right)$ generated by all elements $N(f) y \otimes x-y \otimes M(f) x$, where $f \in \operatorname{Hom}_{\mathscr{C}}(X, Y), x \in M X, y \in N X$ (see [8] and [22]). It is well known that there is a natural isomorphism

$$
\operatorname{Hom}_{Z}\left(N \otimes_{\mathscr{C}} M, L\right)=\operatorname{Hom}_{\mathscr{C} O \mathrm{p}_{-M o d}}\left(N, \operatorname{Hom}_{Z}(M(?), L)\right)
$$

for any abelian group $L$. It follows that $N \otimes_{\mathscr{C}}$ ? and ? $\otimes_{\mathscr{C}} M$ are right exact functors which commute with arbitrary coproducts and direct limits. Moreover, the following isomorphisms hold:

$$
\begin{aligned}
h_{X} \otimes_{\mathscr{C}} M & =M(X), \\
\left(h_{X} / h_{X} I\right) \otimes_{\mathscr{C}} M & =M(X) / I M(X)
\end{aligned}
$$

for each $X \in \mathscr{C}$ and every two-sided ideal $I$ in $\mathscr{C}$.

A $\mathscr{C}$-module $M$ is flat if the functor $? \otimes_{\mathscr{C}} M$ is exact. An easy generalization of the well-known module-theoretic arguments gives the following result:

THEOREM 1.4. A small additive category $\mathscr{C}$ is regular in the sense of von Neumann if and only if every $\mathscr{C}$-module is flat.

Following B. Mitchell, we say that an object $M$ is a continuous well-ordered union of subobjects $M_{\xi}, \xi<\gamma$, where $\gamma$ is an ordinal number if $M_{0}=0, M_{\xi} \subset M_{\tau}$ for $\xi \leqslant \tau$ and $M_{\eta}=\bigcup_{\xi<\eta} M_{\xi}$ wheneyer $\eta$ is a limit ordinal number.

The following lemma is well known (see for instance [16], Lemma 1.4):

LEMMA 1.5. Every infinite directed set $I$ is a continuous well-ordered union of its subobjects $I_{\xi}$ with $\left|I_{\xi}\right|<|I|$.

Now we formulate a result which is repeatedly used in the paper and which may be proved as Theorem 4.2 in [16].

THEOREM 1.6 (Roos). For any direct system $\left\{M_{i}\right\}$ in a Grothendieck category $\mathscr{A}$ and every object $A$ there exists a spectral sequence

$$
E_{2}^{p q}=\lim ^{(p)} \operatorname{Ext}_{\mathscr{A}}^{q}\left(M_{i}, A\right) \Rightarrow \operatorname{Ext}_{\mathscr{A}}^{n}\left(\operatorname{colim} M_{i}, A\right)
$$

where $\lim ^{(p)}$ is the p-th right derived functor of lim (see [16]).

Throughout the paper $\mathscr{A}$ denotes a locally finitely presented Grothendieck category, $\mathscr{C}$ denotes a skeletally small additive category and $R$ is an associative ring with identity. The symbols $\mathrm{lim}$ and colim denote the limit and colimit functor, respectively. The cardinality of a set $I$ is denoted by $|I|$ and $\aleph_{-1}$ denotes any finite cardinal number. A colimit over a directed set will be called a direct limit.
$\S 2$. Purity and flatness. Let $\mathscr{A}$ be a locally finitely presented Grothendieck category and let $\mathrm{mt}$ be a cardinal number. An object $M$ of $\mathscr{A}$ is $\mathrm{m}$-generated (resp. $\mathfrak{m}$-presented) if there exists an exact sequence

$$
L \rightarrow N \rightarrow M \rightarrow 0
$$

where $N$ (resp. $M$ and $L$ ) is a coproduct of 11 copies of finitely presented objects.

THeOrem 2.1. (a) $A \mathscr{C}$-module $M$ is flat and $\mathrm{nt}$-presented iff $M$ is a direct limit of a system of cardinality $\mathrm{nt}$ consisting of finitely generated free $\mathscr{C}$-modules.

(b) If $\mathscr{C}$ has finite coproducts and cokernels, then $\mathscr{C}^{\text {op }}$-module $F$ is flat iff $F$ is a left exact functor.

The first part may be proved as Theorem 1 in [12], and the second as Theorem 1.4 in [8].

It is not difficult to prove the following two lemmas (see [25] and [34]):

LEMMA 2.2. Every $\mathrm{m}$-presented object of $\mathscr{A}$ is a direct limit of a system of cardinality $\mathrm{nt}$ consisting of finitely presented objects.

LEMMA 2.3. An object $M$ is finitely presented iff $\operatorname{Hom}_{\mathscr{A}}(M,-)$ commutes with direct limits (i.e., colimits over directed sets).

An exact sequence

$$
\stackrel{i}{\stackrel{p}{\rightarrow}} \underset{i}{\rightarrow} M \rightarrow 0
$$

is pure if $\operatorname{Hom}_{\mathscr{A}}(X, p)$ is surjective for every finitely presented object $X$. In this case $p$ is called a pure epimorphism and $i$ a pure monomorphism.

Lemma 2.4. (a) If $A \subset B \subset C, A$ is a pure subobject of $C$ and $B / A$ is a pure subobject of $C / A$, then $B$ is a pure subobject of $C$.

(b) $A$ direct limit of pure sequences is pure.

(c) An exact sequence of $\mathscr{C}$-modules $0 \rightarrow A \rightarrow B \rightarrow C \rightarrow 0$ is pure iff the induced homomorphism $M \otimes_{\mathscr{C}} A \rightarrow M \otimes_{\mathscr{C}} B$ is injective for any $\mathscr{C}^{\mathrm{op}}$-module $M$.

(a) and (b) follow immediately from the definitions. (c) is proved in [34].

From Lemma 2.3 immediately follows

COROLLARY 2.5. If $\left\{M_{i}\right\}, i \in I$, is a direct system with I directed, then the natural epimorphism $\oplus M_{i} \rightarrow \operatorname{colim} M_{i}$ is pure.

Recall that an object $M$ is pure-projective (resp. pure-injective) if it is projective (resp. injective) with respect to pure epimorphisms (resp. pure monomorphisms).

It follows from Lemma 2.2 and Corollary 2.5 that a locally finitely presented caregory $\mathscr{A}$ has enough pure-projectives and every its pure-projective object is a summand of a suitable coproduct of finitely presented objects. Moreover, every object $M$ admits a pure-projective resolution, i.e., an exact sequence

$$
\ldots \rightarrow P_{n} \stackrel{d_{n}}{\rightarrow} P_{n-1} \rightarrow \ldots \rightarrow P_{0} \rightarrow M \rightarrow 0
$$

where $P_{i}$ are pure-projective and $\operatorname{Ker} d_{n}$ is a pure subobject of $P_{n}$ for all $n$. Then the pure extension functor Pext may $^{n}$ be defined by

$$
\operatorname{Pext}_{\mathscr{A}}^{n}(M, N)=H^{n} \operatorname{Hom}_{\mathscr{A}}(\boldsymbol{P}, N),
$$


where $\boldsymbol{P}$ is a pure-projective resolution of $M$. The pure-projective and pure-injective dimension we define as

$$
\begin{aligned}
& \text { P.pd } M=\sup \left\{n, \operatorname{Pext}_{\mathscr{A}}^{n}(M, ?) \neq 0\right\}, \\
& \text { P.id } M=\sup \left\{n, \operatorname{Pext}_{\mathscr{A}}^{n}(?, M) \neq 0\right\} .
\end{aligned}
$$

The pure global dimension of $\mathscr{A}$ is defined as

$$
\text { P.gl. } \operatorname{dim} \mathscr{A}=\sup \{\mathbf{P} \cdot \operatorname{pd} M, M \in \mathscr{A}\}=\sup \{\text { P.id } M, M \in \mathscr{A}\} .
$$

Moreover, we set

$$
\text { 1.P.gl. } \operatorname{dim} R=\text { P.gl.dim } R-\operatorname{Mod}
$$

for any ring $R$. The projective dimension of $M$ is denoted by $\operatorname{pd} M$. Finally, the flat dimension of a small additive category $\mathscr{C}$ is

$$
\text { fld } \mathscr{C}=\sup \{\operatorname{pd} M, M \in \mathscr{C}-\mathrm{Fl}\}
$$

where $\mathscr{C}-\mathrm{Fl}$ is the class of all flat $\mathscr{C}$-modules.

It follows from Lemma 2.4 (c) that $\operatorname{pd} M=\mathrm{P}$. pd $M$ for $M \in \mathscr{C}-\mathrm{Fl}$ and therefore

$$
\text { fld } \mathscr{C} \leqslant \text { P.gl. } \operatorname{dim} \mathscr{C} \text {-Mod } .
$$

In the proof of the next theorem we need the following

Proposition 2.6 (Auslander). If a $\mathscr{C}$-module $M$ is a continuous well-ordered union of submodules $M_{\xi}$ such that $\mathrm{pd} M_{\xi+1} / M_{\xi} \leqslant n$ for each $\xi$, then $\mathrm{pd} M \leqslant n$.

Proof. The case $n=0$ follows from [31], Lemma 1 . The inductive step one can obtain in the same way as in [1] by using the functorial natural epimorphism $P(M) \rightarrow M$, where

$$
P(M)=\underset{X \in \mathscr{C}}{\oplus} \underset{f \in M(X)}{\oplus}\left(h^{X}\right)_{f} \quad \text { and } \quad\left(h^{X}\right)_{f}=h^{X} .
$$

THEOREM 2.7 (Jensen [15]). fld $\mathscr{C}=\operatorname{suppd} M$ where $M$ runs over all flat $\mathscr{C}$ - modules for which $\operatorname{pd} M$ is finite.

Proof. Let $s$ denote the right side of the required equality. Since fld $\mathscr{C} \geqslant s$, one can assume that $s$ is finite. Let $M$ be a flat $\mathscr{C}$-module. By Theorem $2.1 M$ is a direct limit of a system $\left\{F_{i}\right\}, i \in I$, with $F_{i}$ free and finitely generated. We shall prove by transfinite induction on $|I|$ that $\mathrm{pd} M$ is finite and therefore not greater than $s$. Clearly this is true if $I$ is finite.

Suppose that $I$ is infinite and consider the exact sequence

$$
0 \rightarrow K \rightarrow \underset{i \in I}{\oplus} F_{i} \rightarrow \underset{i \in I}{\operatorname{colim}} F_{i} \rightarrow 0,
$$

which is pure by Corollary 2.5. By Lemma $1.5 I$ is a continuous well-ordered union of directed subsets $I_{\xi}$ with $\left|I_{\xi}\right|<|I|$ and therefore our sequence is a direct limit of pure sequences

$$
0 \rightarrow K_{\xi} \rightarrow \underset{i \in I_{\xi}}{\oplus} F_{i} \rightarrow \underset{i \in I_{\xi}}{\operatorname{colim}} F_{i} \rightarrow 0
$$

Furthermore, $K$ is a continuous well-ordered union of the pure submodules $K_{\xi}$. Hence $K_{\xi+1} / K_{\xi}$ is flat and therefore pd $K_{\xi+1} / K_{\xi} \leqslant s$ because by the inductive assumption $\mathrm{pd} K_{\xi}$ is finite for each $\xi$. Then by Proposition $2.6 \mathrm{pd} K$ is finite. Thus $\mathrm{pd} M$ is finite and the proof is complete.

Let $L(\mathscr{A})=\mathrm{fp}(\mathscr{A})^{\mathrm{op}}{ }_{-}$Mod where $\mathrm{fp}(\mathscr{A})$ is the full subcategory of $\mathscr{A}$ consisting of all finitely presented objects. Consider a covariant functor

$$
h .: \mathscr{A} \rightarrow L(\mathscr{A})
$$

defined by $h_{A}=\operatorname{Hom}_{\mathscr{A}}(-, A), h_{f}=\operatorname{Hom}_{\mathscr{A}}(-, f)$ for $f \in \operatorname{Hom}_{\mathscr{A}}\left(A, A^{\prime}\right)$. Observe that $h$. commutes with direct limits and arbitrary coproducts. Moreover, a short exact sequence $\boldsymbol{X}$ in $\mathscr{A}$ is pure if and only if the sequence $h_{\mathbf{X}}$ is exact.

THEOREM 2.8. There are natural isomorphisms

$$
\operatorname{Pext}_{\mathscr{A}}^{n}(A, B)=\operatorname{Ext}_{L(\mathscr{A})}^{n}\left(h_{A}, h_{B}\right), \quad n \geqslant 0 \text {. }
$$

Proof. $n=0$. By Lemma $2.2 A=\operatorname{colim} A_{i}$ and $B=\operatorname{colim} B_{j}$ where $A_{i}, B_{j}$ are finitely presented. Then, using the Yoneda Lemma, we get

$$
\begin{aligned}
\operatorname{Pext}_{\mathscr{A}}^{0}(A, B)=\operatorname{Hom}_{\mathscr{A}}(A, B) & =\lim _{i \in I} \operatorname{colim}_{j \in J} \operatorname{Hom}_{\mathscr{A}}\left(A_{i}, B_{j}\right) \\
& =\lim _{i \in I} \operatorname{colim}_{j \in J} \operatorname{Hom}_{L(\mathscr{A})}\left(h_{A_{i}}, h_{B_{j}}\right) \\
& =\operatorname{Hom}_{L(\mathscr{A})}\left(h_{A}, h_{B}\right)=\operatorname{Ext}_{L(\mathscr{A})}^{0}\left(h_{A}, h_{B}\right) .
\end{aligned}
$$

Now suppose that $n>0$ and ler $\boldsymbol{P}$ be a pure-projective resolution of $A$. Then $h_{\boldsymbol{P}}$ is a projective resolution of $h_{A}$ in $L(\mathscr{A})$ and therefore

$$
\operatorname{Pext}_{\mathscr{A}}^{n}(A, B)=H^{n} \operatorname{Hom}_{\mathscr{A}}(\boldsymbol{P}, B)=H^{n} \operatorname{Hom}_{L(\mathscr{A})}\left(h_{\boldsymbol{P}}, h_{B}\right)=\operatorname{Ext}_{L(\mathscr{A})}^{n}\left(h_{A}, h_{B}\right),
$$

which completes the proof.

From Theorems 2.1, 2.8 and Lemma 2.2 we obtain

COROLlary 2.9. The functor $h$. establishes a natural equivalence between $\mathscr{A}$ and the full subcategory $\mathrm{fp}(\mathscr{A})^{\mathrm{op}}-\mathrm{Fl}$ of $L(\mathscr{A})$, which is equal to the full subcategory Lexfp $(\mathscr{A})^{\mathrm{op}}$ of $L(\mathscr{A})$ consisting of all left exact functors.

It is clear that the functor $h$. is left exact and that

$$
R^{n} h \cdot=\operatorname{Ext}_{\mathscr{A}}^{n}(-, \cdot)
$$

where $R^{n} h$. is the $n$th right derived functor of $h$. . Moreover, using Theorem 1.4 and Corollary 2.9 , one can easily prove

THEOREM 2.10. The following conditions are equivalent:

(a) h. is exact.

(b) $\mathscr{A}$ satisfies each of the equivalent conditions in [34], Theorem 4.

(c) $h .: \mathscr{A} \rightarrow L(\mathscr{A})$ is an equivalence of categories.

(d) $\mathscr{A}$ is equivalent to a functor category $\mathscr{C}-\operatorname{Mod}$ where $\mathscr{C}$ is small additive and regular in the sense of von Neumann. 
Remark 1 . The functor $h$. admits a left adjoint which is exact by Ulmer's Flatness Criterion [38]. Then $\mathscr{A}$ is a quotient category of $L(\mathscr{A})$ and $h$. is a section functor (see [10]).

Remark 2. In our situation $\mathrm{fp}(\mathscr{A})^{\mathrm{op}}$-Fl is a Grothendieck category. It would be interesting to give a characterization of those small additive categories $\mathscr{C}$ for which $\mathscr{C}-\mathrm{Fl}$ is a Grothendieck category.

From Theorem 2.8 and Corollary 2.9 we obtain

Corollary 2.11. (a) P.pd $A=\operatorname{pd} h_{A}$ for every object $A$ in $\mathscr{A}$.

(b) P.gl.dim $\mathscr{A}=$ fldfp $(\mathscr{A})^{\mathrm{op}}$.

An immediate consequence of the results of this section and Theorem 1.6 is the following

THEOREM 2.12. (a) If $\left\{M_{i}\right\}, i \in I$, is a direct system in $\mathscr{A}$, then for any object $A$ in $\mathscr{A}$ there exists a spectral sequence

$$
E_{2}^{p q}=\lim ^{(p)} \operatorname{Pext}_{\mathscr{A}}^{q}\left(M_{i}, A\right) \underset{p}{\Rightarrow} \operatorname{Pext}_{\mathscr{A}}^{n}\left(\operatorname{colim} M_{i}, A\right) .
$$

(b) If $|I| \leqslant \kappa_{n}$ then P.pd colim $M_{i} \leqslant 1+n+\operatorname{supP}$.pd $M_{i}$.

(c) P.pd $M \leqslant n+1$ whenever $M$ is $\kappa_{n}$-presented. In particular, pd $M \leqslant n+1$ if $M$ is an $\aleph_{n}$-presented flat $\mathscr{C}$-module.

(d) If $M$ is a continuous well-ordered union of pure subobjects $M_{\xi}$ in $\mathscr{A}$, then P.pd $M \leqslant \operatorname{supP}$.pd $M_{\xi+1} / M_{\xi}^{\prime}$.

(e) P.gl.dim $\mathscr{A}=\operatorname{supP} . \operatorname{pd} M$ where $M$ runs over all objects in $\mathscr{A}$ for which P.pd $M$ is finite

THEOREM 2.13. P.gl.dim $\mathscr{A}=\operatorname{supP}$.id $F$ where $F$ runs over all pure-projective objects of $\mathscr{A}$.

Pro of. Denote by $d$ the right side of the required equality. Since P.gl.dim $\mathscr{A} \geqslant d$, one can assume that $d$ is finite. In virtue of Theorem 2.12(e) it is sufficient to prove that $m=\mathrm{P} . \mathrm{pd} \mathscr{A} \leqslant d$ whenever $m$ is finite. Let

$$
0 \rightarrow P_{m} \rightarrow P_{m-1} \rightarrow \ldots \rightarrow P_{0} \rightarrow A \rightarrow 0
$$

be a pure-projective resolution of $A$ and suppose that $d<m$. Then

$$
\operatorname{Pext}_{\mathscr{A}}^{1}\left(P_{m-1} / P_{m}, P_{m}\right)=\operatorname{Pext}_{\mathscr{A}}^{m}\left(A, P_{m}\right)
$$

and the second member is zero by our assumption. Consequently, the sequence

$$
0 \rightarrow P_{m} \rightarrow P_{m-1} \rightarrow P_{m-1} / P_{m} \rightarrow 0
$$

splits, which contradicts the assumption $m=\operatorname{P} \cdot \operatorname{pd} A$. Then $m \leqslant d$ and the theorem is proved.

Applying the above arguments and using [15], Corollary 4, we also obtain Corollary 2.14. If $R$ is a ring for which w.gl. $\operatorname{dim} R$ is finite, then 1.gl. $\operatorname{dim} R$ $=$ supinj. $\operatorname{dim} P$ where $P$ runs through all projective left $R$-modules.

In the next section the following result is needed:
LEMMA 2.15. Let $\mathscr{B}$ be a full subcategory of $\mathscr{A}$ closed under pure subobjects and pure epimorphic images, and let $\mathscr{F}$ be a family of objects in $\mathscr{B}$ such that every non-zero object of $\mathscr{B}$ has a non-zero pure subobject from $\mathscr{F}$. Then

(a) Each object $B$ of $\mathscr{B}$ is a continuous well-ordered union of pure subobjects $B_{\xi}$ such that $B_{\xi+1} / B_{\xi} \in F$ for each $\xi$.

(b) P.pd $B \leqslant \sup \left\{\mathrm{P} . \mathrm{pd}_{\mathscr{A}} F, F \in \mathscr{F}\right\}$ for any object $B$ of $\mathscr{B}$.

Proof. (b) follows from (a) and Theorem 2.12(d). To prove (a) we put $B_{0}=0$ and $B_{\eta}=\bigcup B_{\xi}$ if $\eta$ is a limit number and $B_{\xi}, \xi<\eta$, are defined. If $B_{\xi}$ is defined and $B / B_{\xi} \neq 0$, we choose a non-zero pure subobject $X \in \mathscr{F}$ of $B / B_{\xi}$ and put $B_{\xi+1}=p^{-1}(X)$ where $p: B \rightarrow B / B_{\xi}$ is the natural epimorphism. From [9] 2.52 and 2.54 and Lemma 2.4.(a) it follows that the embeddings $B_{\xi} \subset B_{\xi+1} \subset B$ are pure and that $B_{\xi+1} / B_{\xi}=X$. This completes the proof.

The above lemma will be applied in the following two cases: if $\mathscr{B}=\mathscr{A}$ and if $\mathscr{A}=\mathscr{C}-\mathrm{Mod}, \mathscr{B}=\mathscr{C}-\mathrm{Fl}$.

\section{\& 3. Generalized stable and factorizable systems.}

DEFINITION 3.1. Let $m$ be an infinite cardinal number or $\aleph_{-1}$ and let $I$ be a directed set. A direct (resp. inverse) system $\left(M_{i}, h_{i j}\right)_{i, j \in I}$ is $m$-factorizable if for every $i \in I$ there exists a set $J_{i} \subseteq\{j \in I, i \geqslant i\}$ of cardinality not greater than $\mathfrak{m}$ and such that each morphism $h_{k i}, k \geqslant i$, admits a factorization

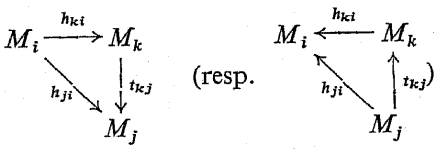

with $j \in J_{i} .\left(M_{i}, h_{i j}\right)_{i, j \in I}$ is $m-$ stable if for any $i \in I$ the directed set $\left\{\operatorname{Ker} h_{j i}\right\}_{j \geqslant i}$ (resp. $\left\{\operatorname{Im} h_{j i}\right\}_{j \geqslant i}$ ) has a cofinal subset of cardinality not greater than $\mathrm{m}$.

$s_{-1}$-factorizable and $s_{-1}$-stable systems will be called shortly factorizable and stable, respectively. In this case one can assume that each set $J_{i}$ has exactly one element. Moreover, $\left(M_{i}, h_{i j}\right)$ is stable iff for any $i$ there is an $j \geqslant i$ such that $\operatorname{Ker} h_{j i}=\operatorname{Ker} h_{k i}\left(\right.$ resp. $\operatorname{Im} h_{j i}=\operatorname{Im} h_{k i}$ ) for all $k \geqslant j$ (see [32]).

Observe that an inverse system of left $R$-modules

$$
\stackrel{\cdot r_{1}}{r^{\cdot r_{2}}} R \leftarrow R \leftarrow R \leftarrow \ldots
$$

is stable iff for each $n$ the chain $\left\{R r_{n+i} \ldots r_{n+1} r_{n}\right\}$ of principal left ideals terminates.

Proposition 3.2 (Laudal). If $F$ is a countable stable inverse system in a Grothendieck category $\mathscr{B}$, then $\lim ^{(1)} F=0$.

The proposition was proved in [20] for $\mathscr{B}=R$-Mod but the proof works in the general case.

Observe that every $\mathrm{m}$-factorizable system is $\mathrm{m}$-stable. Moreover, it is easy to prove the following 
LEMMA 3.3. Every $\mathrm{m}$-stable inverse system consisting of projective objects is itt-factorizable.

The next proposition plays an important role in our further considerations.

Proposition 3.4. Let $\boldsymbol{F}_{I}=\left(F_{i}, f_{i j}\right)_{i, j \in I}$ be an $\mathrm{m}$-factorizable direct system in $\mathscr{A}$ where $F_{i}$ are pure-projective. Then $M=\operatorname{colim} F_{I}$ is an $\mathrm{m}$-directed union (resp. $\aleph_{0}$-directed union if $\mathrm{m}=\mathrm{N}_{-1}$ ) of pure subobjects of the form $N=\operatorname{colim} F_{J}$ where $J$ is $a$ directed subset of $I$ of cardinality $\leqslant \mathfrak{m}\left(\right.$ resp. $\left.\mathrm{s}_{0}\right)$. If $\mathfrak{n}=\mathrm{s}_{n}, n \geqslant-1$, then P.pd $N \leqslant n+1$.

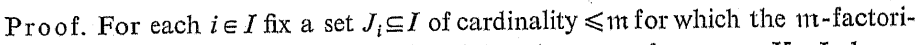
zability condition in Defini:ion 3.1 is satisfied. Furthermore, for every $X \subseteq I$ choose a directed subset $\hat{X}$ such that $|\hat{X}|=|X|$ and $X \subseteq \hat{X} \subseteq I$ (see [2], Lemma 1).

Let $N_{0}$ be an $\mathrm{m}$-generated (resp. countably generated) subobject of $M$ and let $t: \oplus F_{i} \rightarrow M$ be the natural epimorphism. Then $N_{0} \subseteq t\left(\oplus F_{i}\right)$ for some $I_{0} \subseteq I$ with

$\left|I_{0}\right| \leqslant m$ (resp. $\left|I_{0}\right| \leqslant \aleph_{0}$ ). Let $J$ be the union of the sequence $L_{0} \subseteq L_{1} \subseteq L_{2} \subseteq \ldots$ defined by

$$
L_{0}=I_{0}, \quad L_{n+1}=\bigcup_{i \in L_{n}} J_{i} \cup \hat{L}_{n} .
$$

It is easy to verify that
(i) $J$ is directed,
(ii) if $j \in J$ then $J_{j} \subseteq J$,
(iii) $|J| \leqslant\left\{\begin{array}{l}m \text { if } m \text { is infinite, } \\ s_{0} \text { if } m=s_{-1} .\end{array}\right.$

If we put $N=\operatorname{colim} \boldsymbol{F}_{J}$ and denote by $u: N \rightarrow M$ the natural limit morphism, then $N_{0} \subseteq \operatorname{Im} u$. The proof will be finished if we show

$1^{0}$ P.pd $N \leqslant n+1$ whenever $\mathrm{m}=\kappa_{n}, n \geqslant-1$,

$2^{0} u$ is a pure monomorphism.

If $n \geqslant 0,1^{\circ}$ follows from Theorem 2.12(b). Assume $n=-1$ and consider the spectral sequence from Theorem 2.12 for the system $\boldsymbol{F}_{J}$. Since $F_{i}$ are pure-projective, we get an isomorphism

$$
\operatorname{Pext}_{\mathscr{A}}^{1}(N, X)=\lim _{j \in J}^{(1)} \operatorname{Hom}_{\mathscr{A}}\left(F_{j}, X\right)
$$

for any object $X$ of $\mathscr{A}$. By(ii) $F_{J}$ is factorizable, and so the inverse system $\mathrm{Hom}_{\mathscr{A}}\left(\boldsymbol{F}_{J}, X\right)$ is stable. Then by Proposition 3.2 the right member in the above isomorphism is zero, proving that $N$ is pure-projective.

In virtue of Corollary 2.9 , in order to prove $2^{\circ}$ it is sufficient to show that $h_{u}: h_{N} \rightarrow h_{M}$ is a pure monomorphism in $L(\mathscr{A})$ or, equivalently, that the induced group homomorphism $B \otimes_{\mathscr{C}} h_{N} \rightarrow B \otimes_{\mathscr{C}} h_{M}$ is injective for every $\mathscr{C}^{\circ \mathrm{p}}$-module $B$, where $\mathscr{C}=\mathrm{fp}(\mathscr{A})^{\mathrm{op}}$. But from the $\mathrm{m}$-factorizability condition we conclude that

$$
\bigcup_{k \geqslant j} \operatorname{Ker} B \otimes h_{f_{k j}}=\bigcup_{k \in J_{j}} \operatorname{Ker} B \otimes h_{f_{k j}} .
$$

Then in virtue of (ii) the required result follows from
LEMMA 3.5. Let $\left(G, g_{i}\right)=\operatorname{colim}\left(G_{i}, g_{i j}\right)_{i, j \in I}$ in $\mathscr{A}$ and let $J \subseteq I$ be a directed set such that for every $j \in J$

$$
\bigcup_{k \geqslant j} \operatorname{Ker} g_{k j}=\bigcup_{k \in J_{j}} \operatorname{Ker} g_{k j}
$$

where $J_{j}$ is a subset of $J$. If $\left(G^{\prime}, g_{j}^{\prime}\right)=\operatorname{colim}\left(G_{i}, g_{i j}\right)_{i, j \in J}$, then the natural morphism $\varphi: G^{\prime} \rightarrow G$ is injective.

Proof. Since $\mathscr{A}$ is locally finitely presented, it is sufficient to show that every morphism $f: P \rightarrow G^{\prime}$, with $P$ finitely presented, is zero whenever $\varphi f$ is zero.

Assume $\varphi f=0$. It follows from Corollary 2.5 that there is a factorization

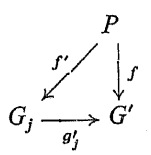

with $j \in J$. Then $0=\varphi g_{j}^{\prime} f^{\prime}=g_{j} f^{\prime}$ and by [25], p. 59, Lemma 8.8, we have

$$
\operatorname{Im} f^{\prime} \subset \operatorname{Ker} g_{j}=\bigcup_{k \geqslant j} \operatorname{Ker} g_{k j}=\bigcup_{k \in J_{j}} \operatorname{Ker} g_{k j}
$$

so $g_{k j} f^{\prime}=0$ for some $k \in J, k \geqslant j$. Hence $f=g_{j}^{\prime} f^{\prime}=g_{k}^{\prime} g_{k j}^{\prime} f^{\prime}=0$ and the lemma is proved.

We say that the category $\mathscr{A}$ is locally $\mathrm{it}$-noetherian if every subobject of a finitely presented object is $\mathrm{t}$-generated.

THEOREM 3.6. Let $\left(M, f_{i}\right)=\operatorname{colim}\left(F_{i}, f_{i j}\right)_{i, j \in I}$ where $F_{i}$ are finitely presented, and let $\mathrm{m}$ be an infinite cardinal number. If $A$ is locally $\mathfrak{n}$-noetherian, $\mathfrak{n} \leqslant \mathrm{m}$, then $\left(F_{i}, f_{i j}\right)$ is $\mathrm{m}$-factorizable if and only if $M$ is an $\mathrm{m}$-directed union of $\mathrm{mt}$-presented pure subobjects.

Proof. The "only if" part follows from Proposition 3.4 because there is an exact sequence (in the notation of 3.4 )

$$
\underset{j_{0} \leqslant j_{1}}{\oplus} F_{j_{0} j_{1}} \rightarrow \underset{j \in J}{\oplus} F_{j} \rightarrow N \rightarrow 0
$$

with $F_{j_{0} j_{1}}=F_{j_{0}}$ (see [16], p. 33). To prove the "if" part observe first that every set $I_{0} \subset I$ with $\left|I_{0}\right| \leqslant \mathfrak{m}$ can be embedded into a directed set $J \subset I$ such that the condition in Lemma 3.5 is satisfied and $|J| \leqslant m$. Indeed, by the $\mathfrak{n}$-noetherian condition, for every $i \in I_{0}$ there is an $L_{i} \subset I$ with $\left|L_{i}\right| \leqslant n$ such that

$$
\bigcup_{j \geqslant i} \operatorname{Ker} f_{j i}=\bigcup_{j \in L_{i}} \operatorname{Ker} f_{j i}
$$

Put $I_{0}^{\prime}=\bigcup_{i \in I_{0}} L_{i}$ and $I_{1}=\hat{I}_{0}$ (in the notation of the proof of 3.4). Continuing in this way, we define a sequence of sets $I_{0} \subset I_{1} \subset I_{2} \subset \ldots$ It is easy to see that their union satisfies the required conditions.

Now consider the natural epimorphism $p: \underset{i \in I}{\oplus} F_{i} \rightarrow M$. Fix $i$ and choose an 
m-generated pure subobject $N_{0}$ of $M$ such that $p\left(F_{i}\right) \subset N_{0}$. It is clear that there exists a directed set $I_{0} \subset\{j \in I, j \geqslant i\}$ satisfying the condition in Lemma 3.5 and such that $N_{0} \subset p\left(\underset{i \in I_{0}}{\oplus} F_{i}\right),\left|I_{0}\right| \leqslant \mathfrak{m}$. Continuing in this way, we define a sequence $I_{0} \subset I_{1} \subset I_{2} \subset \ldots \subset\{j, j \geqslant i\}$ of sets with $\left|I_{n}\right| \leqslant m$ and a sequence $N_{0} \subset N_{1} \subset N_{2} \subset \ldots$ of m-generated pure subobjects of $M$ such that $N_{n} \subset p\left(\underset{i \in I_{n}}{\oplus} F_{i}\right) \subset N_{n+1}, J_{i}=\bigcup_{n=0}^{\infty} I_{n}$ satisfies the condition in Lemma 3.5, $\left|J_{i}\right| \leqslant m$ and $N=p\left(\underset{j \in J_{i}}{\oplus} F_{j}\right)$ is a pure subobject of $M$. We shall show that the $m$-factorizability condition in Definition 3.1 is satisfied.

Let $\left(M^{\prime}, f_{j}^{\prime}\right)=\operatorname{colim}\left(F_{i^{\prime}}, f_{i^{\prime} j^{\prime}}\right)_{i^{\prime}, j^{\prime} \in J_{i}}$. By Lemma 3.5 the natural colimit morphism $\varphi: M^{\prime} \rightarrow M$ is injective and therefore it is a pure monomorphism because $\operatorname{Im} \varphi=N$. For any $k \geqslant i$ consider a commutative diagram

$$
0 \rightarrow M^{\prime} \stackrel{\varphi}{\longrightarrow} M \longrightarrow M / M^{\prime} \rightarrow 0
$$

Since Coker $f_{k i}$ is finitely presented, there exists a morphism $v$ such that $f_{i}^{\prime}=v f_{k i}$ Moreover, by Corollary 2.5 we have a factorization

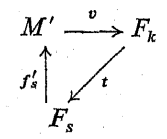

with $s \in J_{i}, s \geqslant i$. Hence $\varphi f_{s}^{\prime}\left(t f_{k i}-f_{s i}\right)=0$, and so $\operatorname{Im}\left(t f_{k i}-f_{s i}\right) \subset \operatorname{Ker} f_{s}^{\prime}$ because $\varphi$ is a monomorphism. Since $F_{i}$ is of finite type, we conclude from [25], p. 59, Lemma 8.8, that $f_{j s}\left(t f_{k i}-f_{s i}\right)=0$ for an $j \in J_{i}, j \geqslant s$, which completes the proof.

COROLLARY 3.7. If $R$ is a commutative ring which has at most $\aleph_{n}, n \geqslant 0$, principal ideals, then fld $R \leqslant n+1$.

Proof. Let $I_{k}, k \in K,|K| \leqslant \aleph_{n}$, be the set of all finitely generated ideals in $R$ and let $\left(M, f_{i}\right)=\operatorname{colim}\left(F_{i}, f_{i j}\right)_{i, j \in I}$ be a non-zero flat $R$-module where $F_{i}$ are finitely generated free. In virtue of Theorem 2.12(c) and Lemma 2.15 it is sufficient to show that $M$ contains a pure $s_{n}$-presented non-zero submodule $N$. Let $f_{i_{0}} \neq 0$. Since $|K| \leqslant \kappa_{n}$ and $R$ is $\kappa_{n}$-notherian, using the same type of arguments as in the first part of the proof of Theorem 3.6 one can show that there exists a directed subset $J$ of $I$, with $|J| \leqslant \aleph_{n}$ and $i_{0} \in J$, such that the condition in Lemma 3.5 is satisfied for each system $\left(R / I_{k} \otimes_{R} F_{i}, R / I_{k} \otimes_{R} f_{i j}\right)_{i, j \in I}, k \in K$.

Put $N=\operatorname{colim}_{j \in J} F_{j}$ and let $\varphi: N \rightarrow M$ be the natural colimit homomorphism. It is clear that $N \neq 0$ is $\kappa_{n}$-presented and by Lemma $3.5 R / I_{k} \otimes_{R} \varphi$ is injective for each $k \in K$. Consequently, $\varphi$ is injective and $\operatorname{Tor}_{1}^{R}\left(R / I_{k}, M / \operatorname{Im} \varphi\right)=0$ for any $k \in K$, so $M / \operatorname{Im} \varphi$ is flat. Thus $\varphi$ is a pure monomorphism and the proof is complete.

COROLLARY 3.8. If $R$ is right coherent, right $s_{n}$-noetherian and right self-FP injective (see [35]), then r.fld $R \leqslant n+1$.
Proof. Let $M=\operatorname{colim}\left(F_{i}, f_{i j}\right)$ be a flat right $R$-module where $F_{i}$ are finitely generated free. Since $R$ is right $\aleph_{n}$-noetherian and coherent then $\left(F_{i}, f_{i j}\right)$ is $\aleph_{n}$-stable, and therefore it is $\aleph_{n}$-factorizable by the self-FP-injectivity of $R$. Then, combining Proposition 3.4 and Lemma 2.15, we obtain the corollary.

Definition 3.9. An object is $1 \mathrm{tt}$-coperfect if any decreasing directed family of its subobjects of finite type has a cofinal subfamily of cardinality at most $\mathrm{mt}$.

$\aleph_{-1}$-coperfect objects are coperfect in the sense of Roos [28] (see also [4] and [5]). A ring $R$ is said to be right $m$-coperfect if it is $m$-coperfect as a right module over itself.

Proposition 3.10. If $P$ is a projective $\mathrm{m}$-coperfect object in $\mathscr{A}$ of finite type, then the endomorphism $\operatorname{ring} E=\operatorname{End}_{\mathscr{A}} P$ is right $\mathrm{m}$-coperfect.

Proof. Let $I_{i}=f_{i 1} E+\ldots+f_{i n_{i}} E, f_{i j} \in E, i, j \in J$, be a decreasing directed family of finitely generated right ideals in $E$. Consider the natural coproduct morphism

$$
\psi_{i}=\left(f_{i 1}, \ldots, f_{i n_{i}}\right): P^{\left(n_{i}\right)} \rightarrow P
$$

where $P^{\left(n_{i}\right)}$ denotes a coproduct of $n_{i}$ copies of $P$. It is clear that $j \geqslant i$ implies $\operatorname{Im} \psi_{j} \subset \operatorname{Im} \psi_{i}$ and by our assumptions there exists a set $J_{0} \subset J,\left|J_{0}\right| \leqslant m$, such that each $\operatorname{Im} \psi_{i}, i \in J$, contains an $\operatorname{Im} \psi_{k}, k \in J_{0}$. By the projectivity of $P, \psi_{k}=\psi_{i} \psi$ for some $\psi$ and hence we conclude that $I_{k} \subset I_{i}$, which proves that the family $I_{k}$, $k \in J_{0}$, is cofinal in $I_{j}, j \in J$.

The next theorem contains a relation between $s_{n}$-coperfectness and a projective dimension of flat modules, and generalizes a result in [3] and [30] (see also [14], Proposition 2.1).

THEOREM 3.11. If every finitely generated free $\mathscr{C}^{\circ \mathrm{p}}$-module is $\aleph_{n}$-coperfect, then fld $\mathscr{C} \leqslant n+1, n \geqslant-1$.

Proof. Consider a contravariant functor

$$
(-)^{d}: \mathscr{C}-\mathrm{Mod} \rightarrow \mathscr{C}^{\mathrm{op}}-\mathrm{Mod}
$$

defined by $(-)^{d}(?)=\operatorname{Hom}_{\mathscr{C} \text {-Mod }}\left(-, h^{?}\right)$ which determines a duality between categories of finitely generated projective $\mathscr{C}$-modules and finitely generated projective $\mathscr{C}^{\mathrm{op}}$-modules.

Let $M$ be a flat $\mathscr{C}$-module. By Theorem 2.1, $M$ can be represented as a direct limit of a system of finitely generated free modules $F_{i}$. It follows from our assumptions and Lemma 3.3 that the dual inverse system of finitely generated free $\mathscr{C}^{\circ \mathrm{p}}$-modules $F_{i}^{d}$ is $\kappa_{n}$-factorizable; hence so is $\left\{F_{i}\right\}$. Then, combining Lemma 2.15 and Proposition 3.4, we obtain the required inequality.

Definition 3.12. A weight of a small category $\mathscr{C}$ is the cardinality $w(\mathscr{C})$ of the disjoint union of sets $\operatorname{Hom}_{\mathscr{C}}\left(X, X^{\prime}\right)$ where $X$ and $X^{\prime}$ run through a fixed set of representatives of isomorphy classes of objects in $\mathscr{C}$.

It is easy to check that every finitely generated free $\mathscr{C}^{\mathrm{op}}$-module is $w(\mathscr{C})$-coperfect whenever $w(\mathscr{C})$ is infinite. Thus Theorem 3.11 yields 2 - Fundamenta Mathematicae XCVI, 
COROLLARY 3.13. If $w(\mathscr{C}) \leqslant \kappa_{n}, n \geqslant 0$, then fld $\mathscr{C} \leqslant n+1$.

Applying Corollary 2.12, we also obtain the following generalization of a result in [14] and [18]:

CoRollary 3.14. If $w(\mathrm{fp}(\mathscr{A})) \leqslant s_{n}, n \geqslant 0$, then P.gl.dim $\mathscr{A} \leqslant n+1$. In particular, r.P.gl. $\operatorname{dim} R \leqslant n+1$ whenever $|R| \leqslant \kappa_{n}, n \geqslant 0$.

For the second statement let us observe that $w(\mathrm{fp}(R-\mathrm{Mod})) \leqslant \max \left(s_{0},|R|\right)$.

DefinItIon 3.15 (see [26], p. 69). $M$ is a Mittag-Leffler object if it is a colimit of a factorizable direct system consisting of finitely presented objects.

The next theorem extends the results of [26], Part $2, \S 2$.

THEOREM 3.16. Let $\boldsymbol{F}_{I}=\left(F_{i}, f_{i j}\right)_{i, j \in I}$ be a direct system consisting of finitely presented $\mathscr{C}$-modules and let $F=\operatorname{colim} F_{I}$. Then the following statements are equivalent:

(a) The direct system $\left(L \otimes_{\mathscr{C}} F_{i}, L \otimes_{\mathscr{B}} f_{i j}\right)$ is stable for any $\mathscr{C}^{\mathrm{op}}$-module $L$.

(b) The inverse system $\left(\operatorname{Hom}_{\mathscr{C}-\operatorname{Mod}}\left(F_{i}, N\right), \operatorname{Hom}_{\mathscr{C}-\text { Mod }}\left(f_{i j}, N\right)\right)$ is stable for any $\mathscr{C}$-module $N$.

(c) $\boldsymbol{F}_{I}$ is factorizable

(d) $F$ is an $s_{0}$-directed union of countably generated pure-projective pure subobjects.

(e) The natural abelian group homomorphism

$$
\Phi:\left(\prod Q_{t}\right) \otimes_{\mathscr{\zeta}} F \rightarrow \prod\left(Q_{t} \otimes_{\varangle} F\right)
$$

is injective for any family $Q_{t}$ of $\mathscr{C}^{\text {op }}$-modules.

(f) $F$ is a Mittag-Leffler object.

Proof. For arbitrary $\mathscr{C}$-modules $M, N$ and each $X \in \mathscr{C}$ we define a homomorphism

$$
t_{X}: N(X)^{*} \otimes_{Z} M(X) \rightarrow \operatorname{Hom}_{\mathscr{C}-\operatorname{Mod}}(M, N)^{*}
$$

setting $t_{X}(f \otimes m)(h)=f h(X) m$ where

$$
-*: \mathscr{C} \text {-Mod } \rightarrow \mathscr{C}^{\mathrm{op}}-\mathrm{Mod}
$$

is a duality functor defined by $M^{*}(-)=\operatorname{Hom}_{Z}(M(-), Q / Z)$ and $Q / Z$ denotes the abelian group of rationals modulo 1 . It is easy to check that $t_{X}$ induce a homomorphism

$$
t: N^{*} \otimes_{\mathscr{C}} M \rightarrow \operatorname{Hom}_{\mathscr{B}-\operatorname{Mod}}(M, N)^{*}
$$

which is an isomorphism whenever $M$ is finitely presented. Since - * is exact and faithful, an inverse system of abelian groups is stable if and only if the dual direct system is stable. From this we infer $(a) \rightarrow(b)$.

(d) $\rightarrow(\mathrm{e})$. Since the tensor product is right exact and $\Phi$ is an isomorphism whenever $F$ is finitely generated free, it is an isomorphism for $F$ finitely presented. Hence $\Phi$ is injective if $F$ is pure-projective and generally if $F$ is a directed union of pure-projectives.
The implication $(\mathrm{c}) \rightarrow(\mathrm{f})$ is trivial and $(\mathrm{f}) \rightarrow(\mathrm{d})$ follows from Proposition 3.4 The proof of $(b) \rightarrow$ (c) and (e) $\rightarrow$ (a) for modules over a ring [26], Part 2, Proposition 2.1.4 and 2.1.5, works in our situation. Thus the proof is complete.

Using the functor $h$., Proposition 3.4 and the above theorem, we obtain the following result, which completes Theorem 3.6 in the case $m=s_{-1}$.

Corollary 3.17. Let $N=\operatorname{colim}\left(P_{i}, p_{i j}\right)$ where $P_{i}$ are finitely presented objects of $\mathscr{A}$. The following statements are equivalent:

(a) The system $\left(P_{i}, p_{i j}\right)$ is factorizable.

(b) $N$ is a Mittag-Leffler object.

(c) $N$ is an $s_{0}$-directed union of countably generated pure-projective pure subobjects.

Corollary 3.18. Let $0 \rightarrow A^{\prime} \rightarrow A \rightarrow A^{\prime \prime} \rightarrow 0$ be a pure exact sequence in $\mathscr{A}$. If $A$ is a Mittag-Leffler object, then so is $A^{\prime}$. If $A^{\prime}$ and $A^{\prime \prime}$ are Mittag-Leffler objects, then $A$ is also a Mittag-Leffler object.

Proof. It is clear that $X$ is a Mittag-Leffler object in $\mathscr{A}$ if and only if $h_{X}$ is a Mittag-Leffler object in $L(\mathscr{A})$. Then, applying Theorem 3.16(e), we obtain the required result.

\$ 4. Pure-injective objects. The aim of this section is to prove the following generalization of a result in [34]:

THEOREM 4.1. Every locally finitely presented Grothendieck category $\mathscr{A}$ has enough pure-injective objects.

The general idea of the proof is due to Kielpiński [17].

Let $M$ be an object in $\mathscr{A}$. An equation scheme over $M$ is a morphism couple

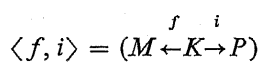

where $i$ is a monomorphism and $P$ is pure-projective. Let $j: M \rightarrow M^{\prime}$ be a monomorphism. A morphism $g: P \rightarrow M^{\prime}$ is called a solution of the equation scheme $\langle f, i\rangle$ in $M^{\prime}$ when $g i=j f$. In this case we say that $\langle f, i\rangle$ is solvable in $M^{\prime}$. An equation scheme $\left\langle f^{\prime}, i^{\prime}\right\rangle$ over $M$ is a subscheme of $\langle f, i\rangle$ if there is a commutative diagram

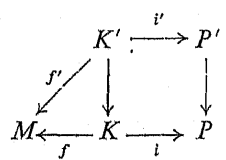

where the vertical arrows are monomorphisms. Finally, we say that the equation scheme $\langle f, i\rangle$ is finitely solvable if its every subscheme $\left\langle f^{\prime}, i^{\prime}\right\rangle$ with $K^{\prime}$ and $P^{\prime}$ of finite type has a solution in $M$.

LEMMA 4.2. An equation scheme $\langle f, i\rangle$ over $M$ in $\mathscr{A}$ is finitely solvable if and only if it is solvable in a pure extension of $M$.

Proof. Suppose that $\langle f, i\rangle$ is finite solvable. Without loss of generality we may assume that $P$ is a coproduct of finitely presented objects. Thus $K$ and $P$ may be 
represented as directed unions of subobjects $K_{t} \subset K$ and $P_{t} \subset P, t \in T$, such that $K_{t}$ is of finite type, $P_{t}$ is finitely presented and $i\left(K_{t}\right) \subset P_{t}$ for all $t$. Let us denote by $f_{t}$ and $i_{t}$ the restrictions of $f$ and $i$ to $K_{t}$, and form a pushout diagram

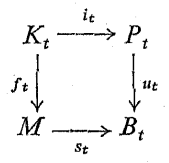

for every $t$ where $s_{t}$ is a split monomorphism by the finite solvability of $\langle f, i\rangle$ The set $T$ is directed if we put

$$
(t \leqslant s) \leftrightarrow\left(K_{t} \subset K_{s} \text { and } P_{t} \subset P_{s}\right) .
$$

Furthermore, there are morphisms $b_{s t}: B_{t} \rightarrow B_{s}$ such that the above diagrams form a direct system. It follows that $s=\operatorname{colim} s_{t}$ is a pure monomorphism and therefore $u=\operatorname{colim} u_{t}$ is the required solution. Since the "if" part is obvious, the proof is complete.

In the proof of the next proposition we shall need the following

LEMMA 4.3. Every object of $\mathscr{A}$ is a directed union of $\mathrm{n}$-generated pure subobjects where $\mathfrak{n}=\max \left(\aleph_{0}, w(f \mathrm{p}(\mathscr{A}))\right)$.

Proof. In view of Proposition 3.4 it is sufficient to show that every direct system $F$ consisting of finitely presented objects of $\mathscr{A}$ is $\mathfrak{n}$-factorizable or, equivalently, that so is the inverse system $h^{F}$ of $\mathrm{fp}(\mathscr{A})$-modules. But this follows from Lemma 3.3 since it is easy to check that every module $h^{X}, X \in \mathrm{fp}(\mathscr{A})$, is $\mathfrak{n}$-stable. alent:

Proposition 4.4. Let $Q$ be an object of $\mathscr{A}$. The following properties are equiv-

(a) $Q$ is pure-injective.

(b) Every finitely solvable equation scheme over $Q$ is solvable in $Q$.

(c) Every finitely solvable equation scheme

$$
\langle f, i\rangle=(\stackrel{f}{\leftarrow \sim} \underset{\leftarrow \rightarrow}{\leftarrow \rightarrow} P)
$$

with $P \mathrm{n}$-generated is solvable in $Q$ where $\mathfrak{n}=\max \left(w(\mathrm{fp}(\mathscr{A})), \mathrm{s}_{0}\right)$.

(d) $\operatorname{Pext}_{s d}^{n}(-, Q)=0$ for $n \geqslant 1$.

Proof. (a) $\rightarrow$ (b) follows from Lemma 4.2, (b) $\rightarrow$ (c) and (d) $\rightarrow$ (a) are obvious. Now we prove (c) $\rightarrow$ (d). Let $X$ be an object of $\mathscr{A}$ and consider a pure exact sequence

$$
\stackrel{i}{i} \underset{X \rightarrow X \rightarrow X}{\rightarrow} \rightarrow 0
$$

with $P$ pure-projective. If $X$ is $\mathfrak{n}$-generated, then $P$ may be chosen $\mathfrak{n}$-generated too, and it follows from (c) that $\operatorname{Pext}_{\alpha A}^{n}(X, Q)=0$ for $n \geqslant 1$. Suppose that $X$ is arbitrary. Using Lemma 4.3, essentially as in the proof of Lemma 2.15, one can show that $P$ is a well-ordered union of pure subobjects $P_{\xi}$ such that $P_{0}=i(K), P_{\eta}=\bigcup \bigcup_{\xi<\eta} P_{\xi}$ if $\eta$ is a limit ordinal number and $P_{\xi+1} / P_{\xi}$ is n-generated for all $\xi$. Since $\operatorname{Pext}_{\mathscr{A}}^{1}(Y, Q)=0$ when $Y$ is $\mathfrak{n t}$-generated, every morphism $K \rightarrow Q$ may be inductively extended to a morphism $P \rightarrow Q$. Hence (d) follows and the proof is complete.

Now, by using Lemma 4.2 and Proposition 4.4, Theorem 4.1 may be proved essentially as Theorem 2 in [17]. Moreover, [36], Proposition 4.5, yields

Corollary 4.5. $\mathscr{A}$ has pure-injective envelopes.

§ 5. Perfect functor categories. Recall that a Grothendieck category $\mathscr{B}$ is said to be coperfect if its every object is coperfect (cf. [28]). $\mathscr{B}$ is perfect if each of its objects admits a projective cover (cf. [3], [6], [19], [32], [39]).

A ring $R$ is right perfect if the category Mod- $R$ of all right $R$-modules is perfect or, equivalently, $R$-Mod is coperfect (see [3] and [4]).

In the next theorem we use the following notations:

$$
\begin{aligned}
\stackrel{r}{r} & \stackrel{r}{r_{1} \cdot} \cdot r_{2} \cdot \\
(R, \stackrel{\rightarrow}{\rightarrow}) & =(R \rightarrow R \rightarrow R \rightarrow \ldots), \\
\cdot r & \quad \cdot r_{1} \cdot r_{2} \\
(M, \stackrel{\leftarrow}{\leftarrow}) & =(M \leftarrow M \leftarrow M \leftarrow \ldots), \quad r_{i} \in R,
\end{aligned}
$$

where $M$ is a right $R$-module and $\cdot r$ denotes the right multiplication by $r \in R$.

THEOREM 5.1. The following statements are equivalent:

(a) $R$ is right perfect.

(b) $\lim ^{(1)}(M, \stackrel{r}{\leftarrow})=0$ for any right $R$-module $M$ and any elements $r_{i} \in R$.

(c) $\lim ^{(1)}(F, \stackrel{\cdot r}{\leftarrow})=0$ where $F$ is a countably generated free right $R$-module and $r_{i} \in R$.

Proof. Fix elements $r_{1}, r_{2}, \ldots \in R$ and put $N=\operatorname{colim}(R, \stackrel{r}{\rightarrow})$. The spectral sequence in Theorem 1.6 gives an isomorphism

$$
\lim ^{(1)}(M, \stackrel{\cdot r}{\rightarrow}) \cong \operatorname{Ext}_{R}^{1}(N, M)
$$

for every right $R$-module $M$. Hence, if we assume (a), then $N$ is projective by [3] and - therefore (b) follows. Now assume (c) and consider the exact sequence

$$
\underset{0 \rightarrow F \rightarrow F \rightarrow N \rightarrow 0}{\stackrel{s}{*}}
$$

where $F$ is a free right $R$-module with basis $x_{1}, x_{2}, x_{3}, \ldots$ and $s\left(x_{i}\right)=x_{i}-x_{i+1} r_{i}$. It follows from the above isomorphism that the sequence splits and therefore (a) is a consequence of [3], Lemma 1.3 and Theorem A. Since (b) $\rightarrow(c)$ is trivial, the proof is complete.

COROLLARY 5.2. Let $P$ be a finitely generated projective $\mathscr{C}$-module. If $\operatorname{Ald} \mathscr{C}=0$, then the ring $E=\operatorname{End}_{\mathscr{G}-\mathrm{Mod}} P$ is right perfect.

Proof. Let $F=E \oplus E \oplus \ldots$ Fix $f_{1}, f_{2}, \ldots \in E$ and denote by $N$ the colimit of the direct system

$$
\stackrel{f_{1}}{P_{1} \rightarrow P_{2} \rightarrow \ldots \rightarrow P_{n} \rightarrow P_{n+1} \rightarrow \ldots}
$$


with $P_{i}=P$. Then, using the spectral sequence in Theorem 1.6, we obtain

$$
\begin{aligned}
\lim ^{(1)}(F, \stackrel{\cdot f}{\leftarrow}) & =\lim ^{(1)}\left(\operatorname{Hom}_{\mathscr{C}-\operatorname{Mod}}\left(P, \oplus P_{i}\right), f_{n}^{*}\right) \\
& =\operatorname{Ext}_{\mathscr{C}-\operatorname{Mod}}^{1}\left(N, \oplus P_{i}\right)=0 .
\end{aligned}
$$

The corollary then follows from Theorem 5.1.

Following Bass [3], we say that the Jacobson radical $J(\mathscr{C})$ of $\mathscr{C}$ is left T-nilpotent if for any sequence

$$
C_{1} \leftarrow C_{2} \leftarrow \ldots \leftarrow C_{n} \stackrel{f_{n}}{\leftarrow} C_{n+1} \leftarrow \ldots
$$

with $f_{i} \in J(\mathscr{C})\left(C_{i+1}, C_{i}\right)$ there exists a $k$ such that $f_{1} f_{2} \ldots f_{k}=0$. A right $T$-nilpotence is defined analogously.

Lemma 5.3 (Bass). If $J(\mathscr{C})$ is left $T$-nilpotent, then $M \neq J(\mathscr{C}) M$ for each $\mathscr{C}$-module $M$. In particular, $J(\mathscr{C}) M$ is superfluous in $M$.

Proof. The module theoretic arguments of Bass [3] generalize.

The next result generalizes Theorem A in [3] (see also [19] and [39]).

THEOREM 5.4. Let $\mathscr{C}$ be a small additive category. Then the following conditions are equivalent:

(1) Every (resp. countably presented) flat $\mathscr{C}$-module is projective.

(2) Every (resp. countably presented) flat $\mathscr{C}-$-module is a Mittag-Leffler object.

(3) The natural homomorphism

$$
\left(\prod Q_{t}\right) \otimes_{\mathscr{8}} M \rightarrow \prod\left(Q_{t} \otimes_{\mathscr{8}} M\right)
$$

is injective for any (resp. countably presented) flat $\mathscr{C}$-module $M$ and any family $Q_{t}$ of $\mathscr{C}^{\text {op }}$-modules.

(4) The category $\mathscr{C}^{\circ \mathrm{p}}-\mathrm{Mod}$ is coperfect.

(5) Each countable inverse system in $\mathscr{C}$ is factorizable.

(6) $J(\mathscr{C})$ is left $T$-nilpotent and the endomorphism ring of any finitely generated projective $\mathscr{C}^{\circ}{ }^{\mathrm{p}}$-module is left perfect.

(7) $J(\mathscr{C})$ is left $T$-nilpotent and $\mathscr{C} / J(\mathscr{C})$ is semi-simple.

(8) The category $\mathscr{C}$-Mod is perfect.

(9) Every flat (resp. countably presented flat) $\mathscr{C}$-module has a projective cover. ment ( ).

Proof. Let us denote by $(')$ the countably presented version of the state-

$\left(3^{\prime}\right) \rightarrow(4)$. If $M_{1} \supset M_{2} \supset M_{3} \supset \ldots$ is a sequence of finitely generated $\mathscr{C}^{\mathrm{op}}$-modules, then there exists a commutative diagram

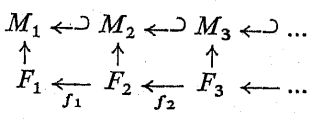

where the vertical arrows are epimorphisms and $F_{i}$ are finitely generated free. Using Theorem 3.16 and the functor $(-)^{d}$ from the proof of Theorem 3.11, we conclude that the bottom row is factorizable. Then $f_{1} f_{2} \ldots f_{n}=f_{1} f_{2} \ldots f_{n+1} g$ for some $g: F_{n} \rightarrow F_{n+1}$, so $M_{n}=M_{n+1}=\ldots$ and (4) follows.

It follows from Theorems 2.1 and 3.16 that $(1) \rightarrow(2) \leftrightarrow(3)$ and $\left(1^{\prime}\right) \leftrightarrow\left(2^{\prime}\right) \leftrightarrow\left(3^{\prime}\right)$. Since $(4) \rightarrow(1)$ is a consequence of Theorem 3.11 for $n=-1$ and $(3) \rightarrow\left(3^{\prime}\right)$ is trivial, all the statements $\left(1^{\prime}\right)-\left(3^{\prime}\right),(1)-(3)$ and (4) are equivalent. Moreover, it follows from Theorem 3.16 and the Yoneda Lemma that $\left(2^{\prime}\right) \rightarrow(5)$, and $(5) \rightarrow(4)$ follows by a simple generalization of Björk's module-theoretic arguments [4], Theorem 2 and [5], §2.

$(5) \rightarrow(6)$. Since the left $T$-nilpotence of $J(\mathscr{C})$ is immediate and we have proved $(4) \leftrightarrow(5)$, (6) follows by using Proposition 3.10 for $\mathfrak{m}=k_{-1}$.

(6) $\rightarrow(7)$ follows from Proposition 1.2 because in view of Lemma 1.1 we have an isomorphism

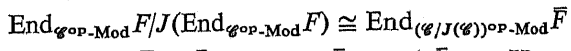

where $F=h_{X_{1}} \oplus \ldots \oplus h_{X_{n}}, \bar{F}=\bar{h}_{X_{1}} \oplus \ldots \oplus \bar{h}_{X_{n}}$ and $\bar{h}_{X_{1}}=\operatorname{Hom}_{\mathscr{\mathscr { C }} / J(\mathscr{G})}\left(-, X_{i}\right)$.

(7) $\rightarrow(8)$. We apply the arguments of Bass [3]. Let $M$ be a $\mathscr{C}$-module. By (7), $M / J(\mathscr{C}) M=\oplus L_{i}^{\prime}$ where $L_{i}^{\prime}$ is a direct summand of an $\bar{h}^{X^{i}}=\operatorname{Hom}_{\mathscr{G} / J(\mathscr{E})}\left(X^{i},-\right)$, $X^{i} \in \mathscr{C}$. Furthermore, it follows from the remark in the proof of Proposition 1.2 that $L_{i}^{\prime}=\operatorname{Im} \bar{h}^{e^{-i}}$ where $\bar{e}^{i}$ is an idempotent in the $\operatorname{ring} E_{i} / J\left(E_{i}\right), E_{i}=\operatorname{End}_{\mathscr{C}} X^{i}$. Since $J(\mathscr{C})$ is left $T$-nilpotent, $J\left(E_{i}\right)$ is a nilideal and therefore $\bar{e}^{i}$ may be lifted to an idempotent $e^{i} \in E_{i}$. Put $L_{i}=\operatorname{Im} h^{e^{i}}$ and consider a commutative diagram

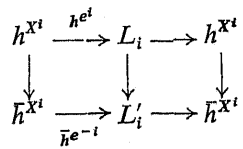

where the middle vertical arrow is an epimorphism. Then we have a commutative diagram

$$
\underset{\oplus \underset{\oplus}{M} \stackrel{h}{\rightarrow} M / J(\mathscr{C}) M}{\uparrow}
$$

where $h$ and $t$ are natural epimorphisms and $h$ is minimal by Lemma 5.3. Hence $t^{\prime}$ is surjective and, since $\operatorname{Ker} t^{\prime} \subset \operatorname{Ker} t=J(\mathscr{C})\left(\oplus L_{i}\right)$, it is minimal and (8) follows. $\left(9^{\prime}\right) \rightarrow\left(1^{\prime}\right)$. We apply the arguments of Mares [21]. Consider an exact sequence of $\mathscr{C}$-modules

$$
\underset{i}{0 \rightarrow K \rightarrow P \rightarrow M \rightarrow 0}
$$

where $M$ is flat, $P$ is projective and $\operatorname{Im} i$ is superfluous in $P$. Assume $K \neq 0$ and choose a finitely generated submodule $K^{\prime} \neq 0$ of $K$. Since $M$ is flat, the sequence in question is pure and therefore there exists a $t: P \rightarrow K$ such that $t i\left(K^{\prime}\right)=K^{\prime}$, so $i\left(K^{\prime}\right) \subset \operatorname{Ker}(1-i t)$. Since

$$
P=\operatorname{Im} i t+\operatorname{Im}(1-i t)=\operatorname{Im} i+\operatorname{Im}(1-i t)
$$

and $\operatorname{Im} i$ is superfluous in $P, 1-i t: P \rightarrow P$ is an epimorphism and therefore $P=P_{1} \otimes \operatorname{Ker}(1-i t)$ with a $P_{1} \subset P$. Thus it follows that $P=P_{1}+\operatorname{Im} i$, so $P=P_{1}$ 
and consequently $\operatorname{Ker}(1-i t)=0$. This is a contradiction. The result is that $K=0$ and therefore $\left(1^{\prime}\right)$ follows.

Since the implications $(8) \rightarrow(9) \rightarrow\left(9^{\prime}\right)$ are trivial, the proof of the theorem is complete.

COROLlary 5.5. If $\mathscr{C}$-Mod is perfect, then any projective $\mathscr{C}$-module is a coproduct of indecomposable cyclic left ideals and any two such decompositions are isomorphic.

Proof. The first statement follows immediately from the proof of Theorem 5.4. The second one is a consequence of the Krull-Remark-Schmidt-Azumaya Theorem because by Corollary 5.2 any indecomposable finitely generated projective $\mathscr{C}$-module has a local endomorphism ring.

We say that the category $\mathscr{A}$ is semiperfect (resp. F-semiperfect) if each of its objects of finite type (resp. finitely presented) has a projective cover (see [19] and [39]). It follows from [3] and [23] that $R$-Mod is semiperfect (resp. $F$-semiperfect) iff $R / J(R)$ is semi-simple (resp. regular in the sense of von Neumann) and idempotents can be lifted modulo $J(R)$. For a functor category we have the following result:

THEOREM 5.6. Let $\mathscr{C}$ be a small additive category. The following statements are equivalent:

(a) $\mathscr{C}$-Mod is semiperfect (resp. F-semiperfect).

(b) $\mathscr{C} / J(\mathscr{C})$ is semi-simple (resp.von Neumann regular) and idempotents of $\mathscr{C} / J(\mathscr{C})$ can be lifted modulo $J(\mathscr{C})$.

(c) Every finitely generated projective $\mathscr{C}$-module has a semiperfect (resp. F-semiperfect) endomorphism ring.

Proof. (b) $\rightarrow$ (a) may be proved as $(7) \rightarrow(8)$ in Theorem 5.4 by using the Nakayama Lemma instead of Lemma 5.3.

(a) $\rightarrow$ (b). The module-theoretic arguments of [3, p. 472] and [23, p. 297] may be carried over verbatim.

(b) $\rightarrow$ (c). If $\mathscr{P}$ is the full subcategory of $\mathscr{C}$-Mod consisting of all finitely generated projective modules, then there is an equivalence $\mathscr{C}$-Mod $=\mathscr{P}^{\text {op }}-$ Mod. Let $P \in \mathscr{P}$. Since End $P=\operatorname{End}_{\mathscr{P} \text { op-Mod }} h_{P}$, in view of the isomorphism from the proof of $(6) \rightarrow(7)$ in Theorem 5.4 (for $\mathscr{C}=\mathscr{P}$ and $F=h_{P}$ ) End $(P) / J($ End $P$ ) is semi-simple (resp. von Neumann regular), and applying (2) to $\mathscr{C}=\mathscr{P}^{\text {op }}$ we conclude that idempotents may be lifted modulo $J($ End $P)$.

$(c) \rightarrow$ (b). By the isomorphism mentioned above every finitely generated free $\mathscr{C} / J(\mathscr{C})$-module $\bar{F}$ has a semi-simple (resp. von Neumann regular) endomorphism ring. Hence by Proposition 1.2 (resp. by the proof of $(\mathrm{c}) \rightarrow(\mathrm{b})$ in Proposition 1.2) $\mathscr{C} / J(\mathscr{C})$ is semi-simple (resp. von Neumann regular). Since the lifting of idempotents modulo $J(\mathscr{C})$ is equivalent to the lifting of idempotents of End $(X) / J($ End $X)$ modulo $J($ End $X)$ for any $X \in \mathscr{C}$, (b) follows and the proof of the theorem is complete.

§ 6. Pure-perfect categories. A pure epimorphism $f: M \rightarrow N$ in $\mathscr{A}$ is purely minimal if a morphism $g: X \rightarrow M$ is a pure epimorphism whenever so is $f g$ or, equivalently,
if $h_{f}$ is a minimal epimorphism in the category $L(\mathscr{A})$.
DeFINITION 6.1. A locally finitely presented category $\mathscr{A}$ is pure-perfect if every object $A$ in $\mathscr{A}$ has a pure-projective cover, i.e. there exists a purely minimal epimorphism $P \rightarrow A$ with $P$ pure-projective.

Now we prove the main result of this section. To formulate it we shall need the following

Definition 6.2. Let $\mathscr{C}$ be an additive category with coproducts. $\mathscr{C}$ is called indecomposably right $T$-nilpotent if for any sequence in $\mathscr{C}$

$$
\underset{1}{\stackrel{f_{1}}{\rightarrow} X_{2} \stackrel{f_{2}}{\rightarrow} X_{3} \rightarrow \ldots}
$$

with $X_{i}$ indecomposable and $X_{i} \not X_{j}$ for $i \neq j$ there is an index $n$ such that $f_{n} f_{n-1} \ldots f_{1}=0$. An indecomposably left $T$-nilpotency is defined analogously.

THEOREM 6.3. Let $\mathscr{A}$ be a locally finitely presented Grothendieck category. The following statements are equivalent:

(1) $\mathscr{A}$ is pure-perfect.

(2) P.gl. $\operatorname{dim} \mathscr{A}=0$.

(3) Every countably presented object of $\mathscr{A}$ is pure-projective.

(4) Every pure-projective object of $\mathscr{A}$ is pure-injective.

(5) Every pure-injective object of $\mathscr{A}$ is pure-projective.

(6) Every object of $\mathscr{A}$ is a Mittag-Leffler object.

(7) The category $L(\mathscr{A})$ is perfect.

(8) The category $\mathrm{fp}(\mathscr{A})$-Mod is locally artinian.

(9) The category $\mathrm{fp}(\mathscr{A})$ is indecomposably right $T$-nilpotent and the endomorphism ring each of its objects is left artinian.

(10) $\mathscr{A}$ is locally noetherian and each of its objects is a coproduct of indecomposable noetherian objects.

Moreover, when $\mathscr{A}=\mathscr{C}$-Mod, each of the conditions (1)-(10) is equivalent to the following statement:

(11) The natural group homomorphism

$$
\left(\prod Q_{t}\right) \otimes_{\mathscr{C}} M \rightarrow \prod\left(Q_{t} \otimes_{\mathscr{C}} M\right)
$$

is injective for any (countably presented) $\mathscr{C}$-module $M$ and any family $Q_{t}$ of $\mathscr{C}^{\circ}{ }^{\mathrm{p}}$-modules.

Proof. In virtue of Corollary 2.11, Lemma 2.2 and Theorem 5.4 applied to $\mathscr{C}=\mathrm{fp}(\mathscr{A})^{\text {op }}$ the statements (1)-(3), (6) and (7) are equivalent. The equivalence $(6) \leftrightarrow(11)$ follows from Theorem 3.16 , and $(2) \leftrightarrow(4)$ is a consequence of Theorem 2.13 .

$(2) \leftrightarrow(10)$. We only have to show $(2) \rightarrow(10)$ since the converse implication is easy. Let $M$ be an object of finite type in $\mathscr{A}$ and $N$ its subobject. Since, as may easily be checked, $M / N$ is finitely presented, $N$ is of finite type and therefore $M$ is noetherian. Hence $\mathscr{A}$ is locally noetherian. Then applying Theorem 5.4 and Corollary 5.5 to $\mathscr{C}=\mathrm{fp}(\mathscr{A})^{\text {op }}$, and using the functor $h .: \mathscr{A} \rightarrow L(\mathscr{A})$, we obtain the required result.

$(7) \rightarrow(8)$. It is easy to check that the category $\mathscr{B}=\mathrm{fp}(\mathscr{A})$-Mod is locally coherent and therefore it is equivalent to the category $\operatorname{Lex} \operatorname{Coh}(\mathscr{B})^{\text {op }}$ of all contravariant left exact functors from $\operatorname{Coh}(\mathscr{B})$ to $\mathrm{Ab}$ where $\operatorname{Coh}(\mathscr{B})$ is the abelian category consisting 
of all coherent object in $\mathscr{B}$ (see [25] and [28], p.204). Furthermore, by Theorem $5.4 \mathscr{B}$ is coperfect and therefore (cf. [28], p. 206) $\mathrm{Coh}(\mathscr{B})$ is an artinian abelian category. Finally, by [25], p. 379, Ex. $7, \mathscr{B}$ is locally artinian.

$(8) \rightarrow(9)$. Let

$$
X_{1} \rightarrow X_{2} \stackrel{f_{2}}{\rightarrow} X_{3} \rightarrow \ldots
$$

be a sequence in $\mathrm{fp}(\mathscr{A})$ with $X_{i}$ indecomposable and $X_{i} \not X_{j}$ for $i \neq j$. In virtue of Theorem 5.4, $J(\mathrm{fp}(\mathscr{A}))$ is right $T$-nilpotent and the endomorphism ring of any $X \in \mathrm{fp}(\mathscr{A})$ is right perfect. Hence $\operatorname{End}_{\mathscr{A}} X_{i}$ is local for all $i$ and, as may easily be checked, $f_{i} \in J(\mathrm{fp}(\mathscr{A}))\left(X_{i}, X_{i+1}\right)$ because $f_{i}$ are non-isomorphisms.

Now let $X \in \mathrm{fp}(\mathscr{A})$. Since $h^{X}$ is a projective and artinian $\mathrm{fp}(\mathscr{A})$-module, by using the same type of arguments as in the proof of Proposition 3:10 one can show that End $h^{X}=(\text { End } X)^{\text {op }}$ is right artinian. Hence the second part of (9) also holds.

$(9) \rightarrow(7)$. It is clear that the category $L(\mathscr{A})$ is equivalent to $\mathscr{C}^{\circ \mathrm{p}}$-Mod where $\mathscr{C}$ is the full subcategory of $\mathrm{fp}(\mathscr{A})$ consisting of representatives of isomorphy classes of indecomposable objects. Then by Theorem 5.4 it is sufficient to show that $J(\mathscr{C})$ is right $T$-nilpotent. Let

$$
\underset{C_{1} \rightarrow C_{2} \rightarrow C_{3} \rightarrow \ldots}{\stackrel{f_{2}}{f_{2}} \rightarrow \ldots}
$$

be a sequence in $J(\mathscr{C})$. If $C_{n}=C_{i}$ for infinitely many indexes $i$, then

$$
\underset{C_{n} \rightarrow C_{n+1}}{\stackrel{f_{n}}{\rightarrow}} \stackrel{f_{n+1}}{\rightarrow} C_{n+2} \rightarrow \ldots
$$

may be considered as a sequence of elements of $J\left(\operatorname{End}_{\mathscr{A}} C_{n}\right)$. Since End $C_{s} C_{n}$ is right perfect, we get $f_{m} \ldots f_{1}=0$ for some $m$. Then without loss of generality we may suppose that $C_{i} \supsetneqq C_{j}$ for $i \neq j$ and by (9) we again have $f_{m} \ldots f_{1}=0$ for some $m$.

(5) $\rightarrow$ (3). Let $M$ be a countably presented object of $\mathscr{A}$. By Theorem $4.1 M$ can be purely embedded in a pure-injective object $Q$ which is pure-projective by our assumption. Then one can suppose that $Q$ is countably presented. It follows from Lemma 2.2 that $Q / M$ is a direct limit of a countable system consisting of finitely presented objects and, according to Theorem 2.12(c), P.pd $Q / M \leqslant 1$. Hence $M$ is pure-projective and (3) is proved.

Since the implication $(2) \rightarrow(5)$ is trivial, the proof of the theorem is complete.

The equivalence of (2), (4) and (5) was proved in [31] and for module categories in [18].

CoRollary 6.4. If $\mathscr{A}$ is locally finite, then P.gl.dim $\mathscr{A}=0$ if and only if $\operatorname{fp}(\mathscr{A})$ is indecomposably right $T$-nilpotent.

Proof. In this case the endomorphism ring of any finitely presented object of $\mathscr{A}$ is semi-primary.

COROLLARY 6.5. 1.P.gl.dim $R=0$ if and only if $R$ is left artinian and the category ${ }_{R}$ fp of all finitely presented left $R$-modules is indecomposably right $T$-nilpotent.

Proof. If 1.P.gl. $\operatorname{dim} R=0$, then $R$ is left noetherian and left perfect, and so it is left artinian.
COROLlary 6.6. If $\mathscr{A}$ is locally finite and there is an upper bound for lengths of indecomposable objects in $\mathrm{fp}(\mathscr{A})$, then P.gl.dim $\mathscr{A}=0$.

In virtue of Corollary 6.4 the corollary is a consequence of the following:

LEMMA 6.7. Suppose that $\mathscr{C}$ is an abelian category in which every object is both noetherian and artinian and let

$$
\underset{M_{1} \rightarrow M_{2} \rightarrow M_{3} \rightarrow \ldots}{f_{2}}
$$

be a sequence of indecomposable objects in $\mathscr{C}$ such that $M_{i} \not M_{j}$ for $i \neq j$. If there is an upper bound for the lengths $l\left(M_{i}\right)$, then $f_{m} \ldots f_{1}=0$ for some $m$.

Proof. Let $l=l(*)=\max _{i} l\left(M_{i}\right)$. It is clear that the lemma holds for $l=1$. If $l>1$ and $l=l\left(M_{i}\right)$ for finitely many indexes $i$, then $(*)$ may be replaced by a sequence $\left(*^{\prime}\right)$ with $l\left(*^{\prime}\right)<l(*)$. Hence without loss of generality we may suppose $l=l\left(M_{i}\right)$ for all $i$. By our assumptions there exist $0=n_{1}<n_{2} \ldots<n_{k}<\ldots$ such that

$$
\operatorname{Ker} f_{n_{k+1}} \ldots f_{n_{k}+1}=\operatorname{Ker} f_{j} \ldots f_{n_{k}+1}, \quad j>n_{k+1},
$$

for $k=1,2,3, \ldots$ Conșider a sequence

$$
\text { (**) } \quad N_{1} \stackrel{g_{1}}{\rightarrow} \stackrel{g_{2}}{\rightarrow} N_{2} \rightarrow N_{3} \rightarrow \ldots
$$

where $N_{k}=M_{n_{k}+1}$ and $g_{k}=f_{n_{k+1}} \ldots f_{n_{k}+1}$. It is clear that $\operatorname{Ker} g_{i}=\operatorname{Ker} g_{i+1} g_{i}$ for all $i$ and therefore

$$
\operatorname{Im} g_{i} \cap \operatorname{Ker} g_{i+1}=0 .
$$

Assume that $f_{m} \ldots f_{1} \neq 0$ for each $m$. Hence $g_{n} \neq 0$ for any $n$ and therefore $l\left(\operatorname{Ker} g_{n}\right)=j_{n}<l$. We shall prove by induction on $j_{n}$ that this is impossible. Clearly it is true for $j_{n}=0$. If $l\left(\operatorname{Ker} g_{n}\right)=j+1$, then $l\left(\operatorname{Im} g_{n}\right)=l-j-1$ and by $(* * *)$ $l\left(\operatorname{Ker} g_{n+1}\right) \leqslant j+1$. Then by the inductive assumption $l\left(\operatorname{Ker} g_{n+1}\right)=j-1$ and again using $(* * *)$ we get a contradiction $\operatorname{Im} g_{n} \oplus \operatorname{Ker} g_{n+1}=N_{n+1}$, which proves the inductive step and completes the proof of the lemma.

COROLlary 6.8. The following conditions are equivalent:

(a) $R$ is left and right artinian and there is an upper bound for lengths of indecomposable objects in ${ }_{R} \mathrm{fp}$ and $\mathrm{fp}_{R}$.

(b) 1.P.gl. $\operatorname{dim} R=0=$ r.P.gl. $\operatorname{dim} R$.

(c) $R$ is left artinian and the set of isomorphy classes of indecomposable finitely presented left $R$-modules is finite.

Proof. The equivalence of (b) and (c) has been proved by Gruson and Jensen (private information). Thus the statement (c) is left-right symmetric and therefore (c) implies (a). Since (a) $\rightarrow$ (b) is a consequence of Corollary 6.6, the proof is complete.

Remark. The implication (a) $\rightarrow$ (c) in the above corollary generalizes the following Brauer-Thrall conjecture, proved by Roiter in [27]: Let $A$ be a finite-dimensional algebra over a field $k$, and suppose that there are infinitely many isomorphy classes of finite-dimensional indecomposable $A$-modules. Then for any $n$ there are indecomposable $A$-modules of finite dimension $\geqslant n$. 
As a consequence of Corollary 6.8 and [7], III, Theorem 5.3, we get

Corollary 6.9. If $R$ is a balanced ring, then 1.P.gl. $\operatorname{dim} R=0=\mathrm{r} . \mathrm{P} . \mathrm{gl}, \operatorname{dim} . R$.

If $R$ is a commutative ring, then by [13], Theorem 4.3, P.gl $\operatorname{dim} R=0$ if and only if each $R$-module is a direct sum of cyclic modules. Now we give an example which shows that a similar result is not true in the non-commutative case.

EXAMPLE ([7], p. 139). Let $D$ be a field and let $\gamma: D \rightarrow D$ be a ring monomorphism such that $\operatorname{dim}_{\gamma(D)} D=2$. Put $R=D \oplus D$ and define a multiplication by $(a, b)(c, d)=(a c, \gamma(a) d+b c)$. It is easy to check that $R$ is a local left and right artinian ring with the Jacobson radical $J(R)=\{(0, b), b \in D\}$, which is simple as a right ideal, and it is a direct sum of two simple left ideals viewied as a left $R$-module. Then $R$ is not quasi-Frobenius and the injective envelope $E\left(R_{R}\right)$ of $R_{R}$ is indecomposable. By [7] $R$ is balanced and hence 1.P.gl. $\operatorname{dim} R=0=$ r.P.gl.dim $R$. Moreover, it follows from [7], Ch. II, that every left $R$-module is a direct sum of cyclic modules. Finally, observe that the finitely presented indecomposable right $R$-module $E\left(R_{R}\right)$ is non-cyclic. In fact, if we assume that $E\left(R_{R}\right)$ is cyclic, then we have a commutative diagram

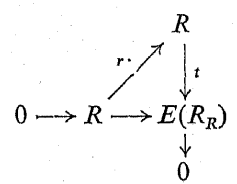

where $r$ is a monomorphism. Since $R$ is local, $r$ is invertible and hence $t$ is an isomorphism. But this is impossible since $R$ is not quasi-Frobenius.

Remark. In [14] a category $D(R)=\mathrm{fp}_{R}$-Mod is applied to an investigation of the pure-injective dimension of left $R$-modules. It is shown that there exists an equivalence of $R$-Mod with the full subcategory of $D(R)$ consisting of $F P$-injective objects (cf. [35]) such that pure-injective left $R$-modules correspond to injective objects in $D(R)$. Recently Gruson and Jensen have proved that 1.P.gl.dim $R=0$ iff $D(R)$ is locally noetherian (see [14]). Thus it follows that the category $R$-Mod is pure-perfect if and only if a direct sum of any family of pure-injective left $R$-modules is pure-injective.

$\S 7$. Final examples. Let $C$ be a coalgebra over a field $k$ and let us denote by $C$-Comod the category of all left $C$-comodules (cf. [37]). It is well known that $C$-Comod is locally finite and that finite-dimensional left $C$-comodules form a set of its generators.

THEOREM 7.1. If $C$ is cocommutative, then P.gl.dim C-Comod $=0$ if and only if $C$ is a direct sum of finite-dimensional coalgebras having a unique composition series of subcoalgebras.

Proof. By [37] $C$ is a direct sum of irreducible coalgebras $C_{i}$. Hence $C$-Comod is equivalent to the product of categories ${ }^{\circ} C_{i}$-Comod and therefore one can assume that $C$ is irreducible. Now, if P.gl. $\operatorname{dim} C$-Comod $=0$, then $C$ is finite-dimensional and therefore $C$-Comod $=C^{*}$-Mod (see [37]). Consequently, the required result is a consequence of [13], Theorem 4.3 .

If $|k| \leqslant s_{n}, n \geqslant 0$, then it is easy to check that $w(\mathrm{fp}(C$-Comod $)) \leqslant \kappa_{n}$, and by Corollary 3.14 we get P.gl. $\operatorname{dim} C$-Comod $\leqslant n+1$. Hence in virtue of Theorem 7.1 we obtain

COROLlaRY 7.2. If $C$ is an infinitely dimensional irreducible cocommutative coalgebra over a countable field, then P.gl. $\operatorname{dim} C$-Comod $=1$.

Now let $\mathscr{H}$ denote the category of all commutative and cocommutative Hopf algebras over a field $k$ and let $\mathscr{D}$ denote its full subcategory consisting of all Dieudonné Hopf algebras (cf. [11]). Then, essentially as above, one can prove that P.gl.dim $\mathscr{H}$ $\leqslant n+1$ and P.gl. $\operatorname{dim} \mathscr{D} \leqslant n+1$ provided $|k| \leqslant \kappa_{n}, n \geqslant 0$. Assume that the characteristic of $k$ is $p>0$ and consider a sequence in the category $\mathscr{D}$

$$
P_{1} \stackrel{f_{1}}{\rightarrow} P_{2} \stackrel{f_{2}}{\rightarrow} P_{3} \rightarrow \ldots
$$

where $P_{n}=k[x] /\left(x^{p^{n}}\right), \Delta(\bar{x})=1 \otimes \bar{x}+\bar{x} \otimes 1, f_{n}(\bar{x})=\bar{x}^{p}$ (see [11]). It is easy to check that this sequence is not factorizable, and so by Corollary 3.17 its colimit $P_{\infty}$ is not pure-projective. Hence, using Theorem 2.12(b), we obtain P.pd ${ }_{\mathscr{H}} P_{\infty}$ $=\mathrm{P} \cdot \mathrm{pd}_{\mathscr{D}} P_{\infty}=1$ and therefore we have the following

COROLlary 7.3. If $k$ is either finite or a countable field of characteristic $p>0$, then P.gl. $\operatorname{dim} \mathscr{H}=$ P.gl. $\operatorname{dim} \mathscr{D}=1$.

A similar result can be obtained for the category of graded abelian Hopf algebras over a perfect field of finite characteristic $p \neq 2$ (cf. [29]).

We do not know if the assumption in Corollaries 7.2 and 7.3 about the cardinality of $k$ is essential.

\section{References}

[1] M. Auslander, On the dimension of modules and algebras III, Nagoya Math. J. 9 (1955), pp. $67-77$.

[2] S. Balcerzyk, On projective dimension of direct limit of modules, Bull. Acad. Polon. Sci. Sér. Sci. Math. Astronom. Phys. 14 (1966), pp. 241-244.

[3] H. Bass, Finitistic dimension and a homological generalization of semi-primary rings, Trans. Amer. Math. Soc. 95 (1960), pp. 466-488.

[4] J. E. Björk, Rings satisfying a minimum condition on principal ideals, J. Reine Angew. Math. 236 (1969), pp. 112-119.

[5] - Radical properties of perfect modules, ibid. 253 (1972), pp. 78-86.

[6] I. Bucur and A. Delea nu, Introduction to the Theory of Categories and Functors, LondonNew York-Sydney 1968.

[7] V. Dla b and C. M. Ringel, Balanced rings, Lecture Notes in Math. 246 (1972), pp. 77-145.

[8] J. Fischer, The tensor product of functors, satellites and derived functors, J. Algebra 8 (1968), pp. 277-294.

[9] P. Freyd, Abelian Categories, New York 1964

[10] P. Gabriel, Des catégories abéliennes, Bull. Soc. Math. France 90 (1962), pp. 323-448.

[11] - Sur les catégories abéliennes localement noetheriennes et leurs applications aux algébres etiudés par Dieudonné, Sem. Serre, 1960. 
[12] V. E. Govorov, On flat modules, Sibirs. Mat. J. 6 (1965), pp. 300-304.

[13] P. Griffith, On the decomposition of modules and generalized left uniserial rings, Math. Ann. 184 (1970), pp. 300-308.

[14] L. Gruson and C. U. Jensen, Modules algébraiquement compact et foncteurs $\varliminf^{(i)}$, C. R. Acad. Sci., Paris, Sér. A 276 (1973), pp. 1651-1653.

[15] C. U. Jensen, On the vanishing of $\varliminf^{(i)}$, J. Algebra 15 (1970), pp. 151-166.

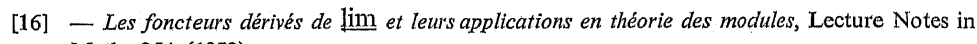
Math. 254 (1972).

[17] R. Kiełpiński, On $\Gamma$-pure injective modules, Bull. Acad. Polon. Sci. Sér. Sci. Math. Astronom. Phys. 15 (1967), pp. 127-131.

[18] - and D. Simson, On pure homological dimension, ibid. 23 (1975), pp, 1-6.

[19] M. Harada, Perfect categories, Osaka J. Math. 10 (1973), pp. 329-368.

[20] O. A. Laudal, Sur la limite projective et la théorie de la dimension, Top. et Géom. Dif., Sém. Ehresmann, 1961, 23 p.

[21] B.E. Mares, Semi-perfect modules, Math. Z. 83 (1963), pp. 347-360.

[22] B. Mitchell, Rings with several objects, Advances in Math. 8 (1972), pp. 1-161.

[23] U. Oberst and H. J. Schneider, Die Struktur von projektiven Moduln, Inventiones Math. 13 (1971), pp. 295-304.

[24] B. L. Osofsky, Upper bound on homological dimension, Nagoya Math. J. 32 (1968), pp. 315 322

[25] N. Popescu, Abelian Categories with Applications to Rings and Modules, London-New York 1973.

[26] M. Raynaud et L. Gruson, Criteres de platitude et de projectivité. Techniques de „platification" d'un module, Inventiones Math. 13 (1971), pp. 1-89.

[27] A. V. Roiter, The unboundedness of the dimension of the indecomposable representations of algebras that have a finite number of indecomposable representations, Izv. Acad. Nauk SSSR 32 (1968), pp. 1275-1282.

[28] J. E. Roos, Locally Noetherian categories and generalized strictly linearly compact rings, Lecture Notes in Math. 92 (1969), pp. 197-277.

[29] C. Schoeller, Etudé de la catégorie des algébres de Hopf commutatives connexes sur un corps, Manuskripta Math. 3 (1970), pp. 133-155.

[30] D. Simson, A remark on projective dimension of flat modules, Math, Ann. 209 (1974), pp. 181182.

[31] - On colimits of injectives in Grothendieck categories, Arkiv för Matematik 12 (1974), pp. 161-165.

[32] - Functor categories in which every flat object is projective, Bull. Acad. Polon. Sci. Sér. Sci. Math. Astronom. Phys. 22 (1974), pp. 375-380.

[33] - Serwantność $i$ wymiary homologiczne $w$ lokalnie skończenie przedstawialnych kategoriach Grothendiecka, Uniwersytet Mikołaja Kopernika, Prace habilitacyjne, Torun 1974.

[34] B. Stenström, Purity in functor categories, J. Algebra 8 (1968), pp. 352-361.

[35] - Coherent rings and FP-injective modules, J. London Math. Soc. 2 (1970), pp. 323-329.

[36] - Pure submodules, Arkiv för Matematik 7 (1967), pp. 159-171.

[37] M. E. Sweedler, Hopf Algebras, New York 1969.

[38] F. Ulmer, A flatness criterion in Grothendieck categories, Inventiones Math. 19 (1973), pp. $331-336$.

[39] M. Weidenfeld et G. Weidenfeld, Idéaux d'rne catégories préadditive, application aux catégories semi-parfaites, C. R. Acad. Sci. Paris, Sêrie A, 270 (1970), pp. 1569-1571.

INSTYTUT MATEMATYKI, UNTWERSYTET MIKOEAJA KOPERNIKA

INSTITUTE OF MATHEMATICS, NICHOLAS COPERNICUS UNIVERSITY

Accepté par la Rédaction le 17. 2. 1975

\section{A locally connected non-movable continuum that fails to separate $E^{3}$}

by

D. R. McMillan, Jr. * (Madison, Wisc.)

Abstract. A locally connected continuum $X$ (Fig. 2) is constructed by tunneling into a 3-cell in such a way that $E^{3}-X$ is connected. The non-movability of $X$ is proven using the three-manifold techniques of Haken and Waldhausen.

1. Introduction. K. Borsuk has introduced and studied the important shape property of movability for compacta. Examples of movable compacta include compact absolute neighborhood retracts and compacta embeddable in 2-manifolds (see [1], [10], and [7]). Some of the more exotic continua, such as solenoids, are not movable ([1]). It seems especially worthwhile to seek convenient characterizations of movable compacta in 3-manifolds. Most examples of locally connected continua in Euclidean 3 -space $E^{3}$ that come to mind are movable (for example, locally connected one-dimensional continuua are movable), but in general this is not enough to do the job. In particular, Borsuk gave in [2] an example of a locally connected, non-movable continuum in $E^{3}$. His example separates $E^{3}$ into two pieces. In an effort to focus on what does (and does not) make a continuum (i.e., compact, connected Hausdorff space) in $E^{3}$ movable, we present an example of a locally connected, non-movable continuum with connected complement in $E^{3}$. (This answers the second part of Borsuk's Problem 5.5 in [2].)

A compactum $X$ is movable if for some (and hence for every) embedding $X \subset Q$ (= the Hilbert cube), the following holds: Each neighborhood $U$ of $X$ contains a neighborhood $V$ of $X$ such that for each neighborhood $W$ of $X$, the final stage of some homotopy of $V$ in $U$ throws $V$ into $W$. Of course, it can be shown that if $X$ lies in a nice space, such as a manifold $M$, then $X$ is movable by the preceding definition if and only if the corresponding movability statement holds for $X$ with respect to its neighborhoods in $M$. Our example is constructed from a 3 -cell by an infinite sequence of tunneling operations. (See Figure 2: any resemblance to a Christmas tree is coincidental.)

While the example itself is easy to describe, our proof of its non-movability seems rather elaborate. Perhaps simpler proofs and/or examples exist. We rely heavily

* Research supported by N. S. F. grant GP-38877A\#1. 